\title{
Receptor Encoding of Moving Tactile Stimuli in Humans. II. The Mean Response of Individual Low-Threshold Mechanoreceptors to Motion Across the Receptive Field
}

\author{
Gregory K. Essick' and Benoni B. Edin ${ }^{2}$ \\ 'Dental Research Center and Curriculum in Neurobiology, University of North Carolina at Chapel Hill, Chapel Hill, North \\ Carolina 27599-7455 and 2Department of Physiology, Umeå University, S-901 87 Umeå, Sweden
}

The mean firing rate evoked in 70 cutaneous, low-threshold mechanoreceptors in the human median, radial, and inferior alveolar nerves by stimulus motion across the skin was quantitatively studied. Moving stimuli, controlled for velocity, direction, and length of skin traversed, were provided by a servo-controlled motor that carried a brush across the receptive field. Each unit was studied with stimuli delivered at multiple velocities from 0.5 to $32 \mathrm{~cm} / \mathrm{sec}$ in at least two opposing directions.

A power function provided an excellent description of the MFR-versus-velocity relationship. The exponent $n$ was interpreted to reflect the receptor's sensitivity to changes in stimulus velocity, and the multiplicative constant $c$, the predicted response to stimuli moving at $1.0 \mathrm{~cm} / \mathrm{sec}$. The fast adapting mechanoreceptors exhibited higher sensitivity to stimulus velocity than the slowly adapting mechanoreceptors. The mean velocity at which the fast adapting units were predicted to first respond to movement was also higher.

Estimates of $n, c$, or both differed significantly for stimuli delivered in opposing directions for more than $70 \%$ of the mechanoreceptors. No direction of motion consistently led to power function parameters with higher values so as to suggest a "preferred" regional direction of motion for the entire population. Neither the directional difference in $n$ nor $c$ could be attributed to directional differences in the forces applied across the receptive fields. These findings suggest that information about velocity and direction is represented in the mean firing rate responses evoked in the population of mechanoreceptors activated by a moving tactile stimulus.

[Key words: human, microneurography, tactile stimuli, receptor encoding, motion, velocity discrimination, directional sensitivity]

Early studies demonstrated that movement across the receptive field is most effective in evoking activity in cutaneous lowthreshold mechanoreccptors (in man and subhuman primates;

Received Apr. 5, 1994; revised July 11, 1994; accepted July 15, 1994.

The neurophysiological experiments were conducted at the Department of Physiology, University of Umea, Sweden. We gratefully acknowledge the technical assistance provided by Mr. Göran Sandstrôm and Mr. Anders Băckström. The work was supported by the Swedish Medical Research Council (Grants 08667 and 00045), the Office of Naval Research, Arlington, VA (Grant No. N00014-90-J1838), the Natiunal Institute of Dental Research (Program Project No. DE07509), and the University of North Carolina School of Dentistry.

Correspondence should be addressed to Benoni B. Edin, M.D., Ph.D., at the above address.

Copyright (C) 1995 Society for Neuroscience $0270-6474 / 95 / 150848-17 \$ 05.00 / 0$
Vallbo and Hagbarth, 1968; Hagbarth et al., 1970; Whitsel et al., 1972; Järvilehto et al., 1976; Vallbo et al., 1979). These studies also suggested that the mean-firing-rate response of individual mechanoreceptors represents information about the velocity and direction of movement. This hypothesis, however, has never been systematically evaluated for human subjects.

To this end, and to better appreciate the contribution of individual afferents to the population-encoding of information about moving tactile stimuli, we quantitatively studied 70 slowly and fast adapting mechanoreceptors in the median, radial, and inferior alveolar nerves of man. Natural movement across the receptive field was provided by a tactile stimulator that moved a small brush across the receptive field at a constant velocity in a specified direction. To determine whether velocityand direction-dependent variability in each neuron's response could be attributed to concomitant velocity- and direction-dependent variability in the forces applied across the receptive field, the brush was equipped with transducers to sample the normal (indenting) and tangential (compressing and stretching) forces applied across the skin.

In the preceding companion paper (Edin et al., 1994), it was demonstrated that many mechanoreceptors (particularly the slowly adapting units) discharge in a remarkably consistent manner upon replication of the same moving brush stimulus to the receptive field. The discharge was shown to not only reflect indentation of the skin, but also lateral skin compression and stretch. Moreover, the mechanoreceptors were shown to display differences to stimuli that move at different velocities and directions and that apply different forces across the receptive fields. The most notable difference to variation in stimulus velocity and direction was shown to be in the intensity of the discharge and spatial discharge pattern, respectively. In response to different forces applied across the receptive field, most mechanoreceptors exhibited changes in discharge intensity or in the degree to which the details of the spatial discharge pattern were made evident. It was argued that the details of the discharge patterns evoked in the population of excited receptors were likely not used by the CNS to infer information about direction and velocity of movement across the skin. Rather, the discharge intensity was presented as a plausible information-bearing attribute of the stimulus-evoked response.

In this paper, we further examine the capacity of the discharge intensity (viz., of the stimulus-cvoked mean firing rate) of individual mechanoreceptors to encode information about stimulus velocity. Emphasis is placed on the identification of variations in the velocity-encoding capacities that can be attributed 
to diffcrences in the units' adaptational properties, the peripheral distributions of the nerves, and the forces applied across the receptive fields. Of secondary emphasis is the impact of changes in the direction of stimulus motion and of the total area of skin contacted by the moving stimuli.

\section{Materials and Methods}

The reader is referred to the companion article for in-depth descriptions of the neurophysiological recording technique, delivery of moving tactile stimuli across the skin, sampling of the forces applied to the receptive field, and the general experimental protocol (Edin et al., 1994). Briefly summarized, single-unit recordings were obtained from mechanoreceptors in the median, radial, and inferior alveolar nerves using the microneurographic technique described by Vallbo and others (Vallbo and Hagbarth, 1968; Johansson and Olsson, 1976; Johansson et al., 1988). After each unit was isolated, calibrated nylon monofilaments were employed to locate the skin position of lowest mechanical threshold and to define the threshold force. The total region of skin over which the unit was responsive to four times the threshold force defined the receptive field (RF). Based on RF size, adaptation properties, presence of spontaneous activity, and regularity of evoked response, all units were classified as SAI, SAII, FAI, FAII, or F $\Lambda$-hair using standard criteria (Knibestöl and Vallbo, 1970; Vallbo and Johansson, 1984; Edin, 1992). All fast adapting units were grouped into a common (fast adapting, "FA") category. This is because only two fast adapting units were not classified as type I, and their responses to moving stimuli were similar to those of the FAI units. A total of 93 cutaneous mechanoreceptors were isolated and classified.

Moving tactile stimuli were delivered across the RF by a brushing stimulator used previously in neurophysiological and psychophysical studies (Whitsel et al., 1972; Essick and Whitsel, 1985a,b; Essick et al., 1988, 1990, 1991a,b). The stimulator device consisted of a servomotor and controlling electronic circuitry. The motor shaft rotated a brush across the skin. Each stimulus trial consisted of a brush movement over a thin Teffon plate taped to the skin. A $1.4 \mathrm{~cm} \times 1.4 \mathrm{~cm}$ aperture in the plate was centered over the RF of the afferent under study and defined the precise area of skin to receive the stimuli. Stimuli were provided by one of four brushes which differed in apparent stiffness and delivered different normal and tangential forces to the skin. Approximately $90 \%$ of the data were obtained with either a brown or relatively stiffer white brush. About $88 \%$ of these data were obtained with the bristles extended $2-3.5 \mathrm{~mm}$ through the aperture. Given these conditions, the brown and white brush typically delivered normal forces of $21.8-67.6 \mathrm{mN}$ and $151-257.2 \mathrm{mN}$, respectively. In addition to differences in force, the area of skin contacted by the two brushes also differed. As a result, the stress applied by the white brush was approximately 4.5 times that applied by the brown brush.

In order to monitor the normal and tangential forces applied to the RF, the mechanical interface between the shaft of the motor and the brush was equipped with strain gauges. To allow accurate timing of the period during which the brush was in contact with the skin, two pairs of infrared light-emitting diodes (IREDs) and fast-responding photosensitive plates were mounted on the edges of the stimulus aperture. For all analyses reported in this article, forces are expressed as values averaged over the period during which the brush bristles contacted only the skin.

An MS-DOS operated 386 stimulus delivery system was used to control stimulus sequence, velocity, and direction. Randomized blocks of moving stimuli were typically delivered at five or seven velocities from 0.5 to 32 centimeters per second $(\mathrm{cm} / \mathrm{sec})$ in each of two opposing directions (clockwise and counterclockwise). Interstimulus intervals were 4-6 sec in length. Blocks of trials were repeated 4-10 times. The data from each series of blocks constituted a run.

After each experimcnt, the individual nerve spikes were inspected using previously described algorithms (Edin et al., 1988). The spike trains were then sorted by velocity and direction for each run. For every spike train (i.e., stimulus trial), values for 48 variables were extracted and written to an ASCII file. These variables included information about the number of stimulus-evoked spikes, mean and peak firing rates, duration of stimulus-evoked activity, spontaneous activity, normal and tangential forces, as well as descriptive information about the unit studied, its RF and conditions of stimulation. Mean firing rate was calculated as the number of stimulus-evoked spikes divided by the duration of the activity. Peak firing rate was defined as the reciprocal of the shortest, stimulus-evoked interspike interval. For units that were spontaneously active, stimulus-evoked firing rates were adjusted by subtraction of the background firing rate.

All statistical analyses were performed on an IBM compatible 486 computer using SAS software (SAS Institute Inc., 1988). For the questions addressed in this article, descriptive statistics and plots of the relevant variables were first examined. Analyses of variance, covariance, or linear regressions were then performed. The residuals of analyses of variance and covariance were checked for normality, and logarithmic transformations of the data were undertaken when indicated. Accordingly, geometric means and standard deviations (rather than arithmetic means and standard deviations) are reported below for values that were distributed log normally. Significant effects were subsequently investigated with $t$ tests and the Ryan-Einot-Gabriel-Welsch multiple range test as dictated by the number of means to be compared.

\section{Results}

The response of each mechanoreceptor to stimulus motion occurring at multiple velocities (median number equaled 7) in each of two opposing directions were obtained. Ten units were, in addition, studied with opposing directions delivered along a second orientation across the RF, orthogonal to the first. Moreover, 16 units were studied with more than one brush or bristle extension against the skin. And 15 units were stimulated through more than one aperture so as to vary the total area of skin exposed to the moving brush. All together, 12,323 trials of single-unit data were collected. Force data were available for 62 of the 70 quantitatively studied units.

\section{Effect of velocity on mean neural response}

The human data analyzed in this article consisted of 152 runs, each of which contained the neural response to multiple velocities for two opposing directions of motion. Most of the RFs of mechanoreceptors in the median and radial nerves were stimulated with seven velocities (six octaves from 0.5 to $32 \mathrm{~cm} / \mathrm{sec}$ ). In contrast, most of the RFs of mechanoreceptors in the inferior alveolar nerve were stimulated with five velocities (viz., those used in previous psychophysical studies of human direction discrimination: $0.5,2,6,12$, and $32 \mathrm{~cm} / \mathrm{sec}$; Essick et al., 1988, 1991 a). Thus, the range of velocities employed in the present study was narrower than those employed in monkey and cat experiments (Whitsel et al., 1972; Young et al., 1978; Franzén et al., 1984; Greenspan, 1992), but encompassed that used by human subjects to explore and discriminate the texture of rigid grooved surfaces (e.g., range $1-25 \mathrm{~cm} / \mathrm{sec}$, Lederman, 1974; mean $11.5-18.5 \mathrm{~cm} / \mathrm{s}$, peak $18-29 \mathrm{~cm} / \mathrm{sec}$, Morley et al., 1983 ; range $2.5-15 \mathrm{~cm} / \mathrm{sec}$, Sinclair and Burton, 1991; see also Meyers et al., 1958, and Lamb, 1983a). Over the velocity range tested, the human data were grossly similar to that reported for the monkey and cat.

As an example, Figure 1 displays spike train raster plots from two runs. The RF of the FAI unit whose data are shown was located on the glabrous, proximal phalanx of the index finger and was stimulated with brush strokes moving proximal-todistally $(\mathrm{P} \rightarrow \mathrm{D})$ and distal-to-proximally $(\mathrm{D} \rightarrow \mathrm{P})$. Figure $1 A$ depicts the first four spike trains cvoked at each velocity and direction by the white brush; Figure $1 B$, by the brown brush. Note that the responsivity of the unit to the white brush was appreciably greater than it was to the brown brush. All data obtained from this unit with stimuli moving in the distal-toproximal direction are graphically summarized in Figure 2. Figure $2 A$ depicts the number of spikes; Figure $2 B$, duration of activity; Figure $2 C$, mean firing rate; and Figure $2 D$, peak firing rate. Note that as velocity increased, the number of stimulus- 

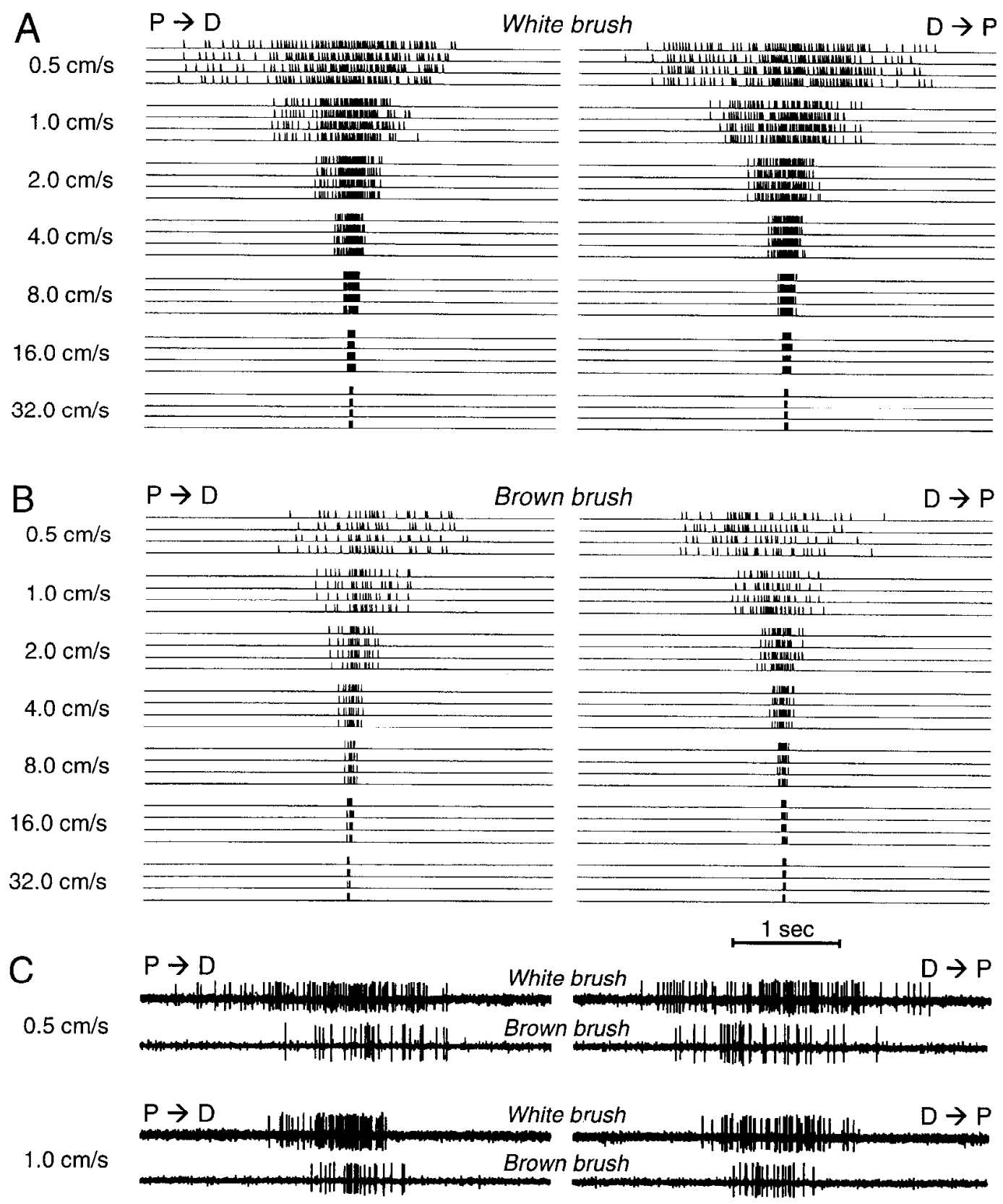

Figure 1. The response of an FAI unit to seven different velocities of stimulus motion. The response to brush strokes moving proximal- to-distally $(P \rightarrow D$; spike trains on the left) and distal-to-proximally $(D \rightarrow P$; spike trains on the right) is shown. The data in $A$ were obtained with the white brush; in $B$, with the brown brush. $C$, Example of actual nerve signals for each direction for each brush for two stimulus velocities.

evoked spikes and duration of stimulus-evoked activity decreased (see Figs. 1, 2A,B). In contrast, as illustrated by Figure $2, C, D$, the mean firing rate and peak firing rate increased. The peak firing rate increased less dramatically and exhibited greater variability than the mean firing rate. The trends illustrated in Figure 2 were typical of all units we studied with one exception: that is, some FA units responded poorly to the lowest velocities of stimulus motion (see Fig. 3). As will be argued in the discussion, these units that responded poorly to the lowest velocities attest to a physiological diversity in the population of fast adapting mechanoreceptors.

The quantitative relationship between the discharge rate of individual cutaneous afferents and the velocity of natural brush- ing stimuli applied across their RFs has been reported for the monkey (Whitsel et al., 1972; Young et al., 1978; Franzén et al., 1984) and cat (Greenspan, 1992) hairy hindlimb. In these studies a power function adequately described the relationship between the discharge rate and the velocity (mean $R^{2}=0.89$ over the velocity range $0.7-75 \mathrm{~cm} / \mathrm{sec}$, Young et al., 1978; mean $R^{2}=0.984$ over the velocity range 1.17 and $50 \mathrm{~cm} / \mathrm{sec}$, Greenspan, 1992). Although Whitsel and colleagues initially characterized the discharge rate of the afferents by their peak firing rate (Whitsel et al., 1972; Young et al., 1978), mean firing rate was later found to be a less noisy and a more sensitive indicator of stimulus velocity (Franzén et al., 1984).

To characterize the impact of variations in stimulus velocity 

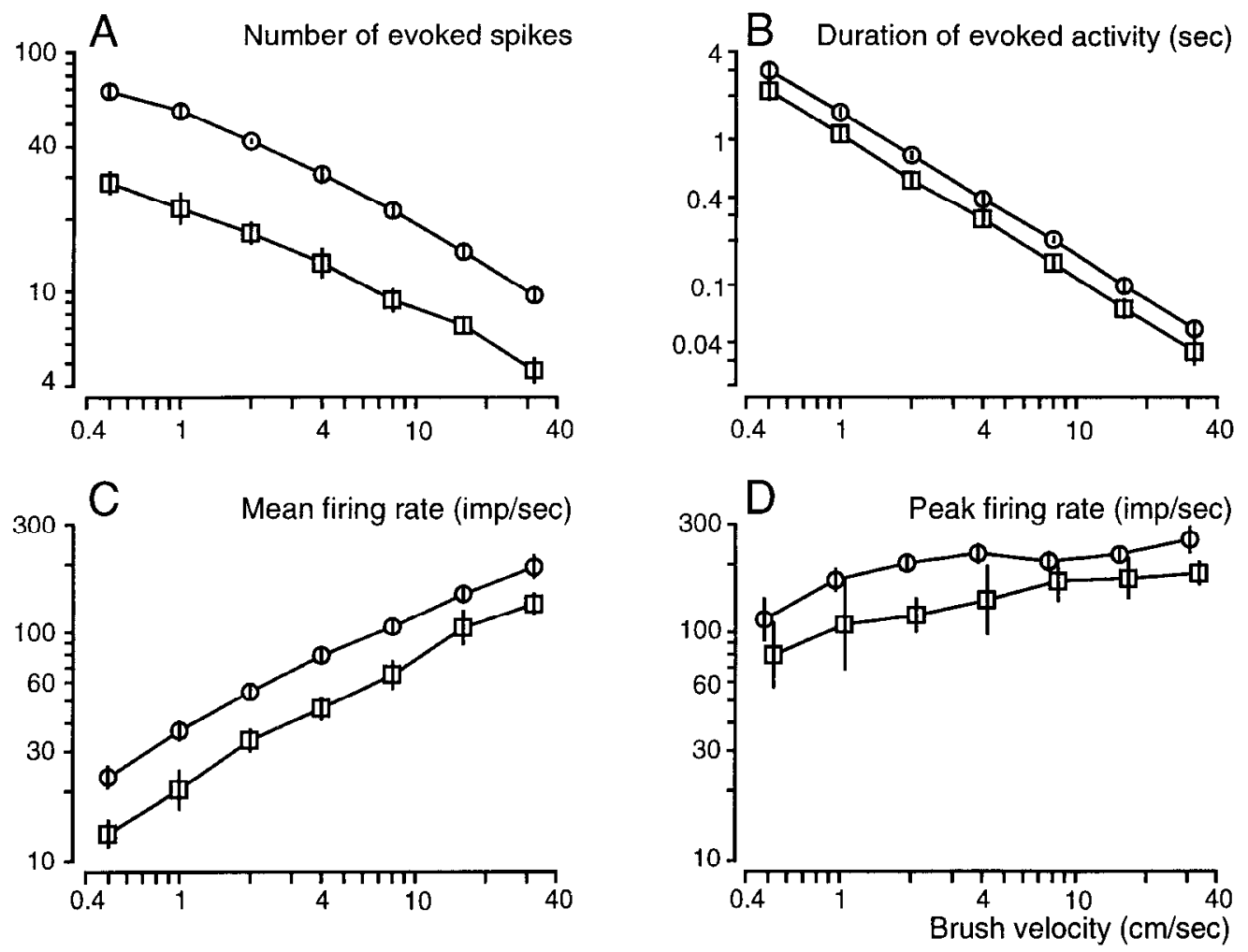

Figure 2. Plots of four measures of the neural response to stimuli moving $\mathrm{D} \rightarrow \mathrm{P}$ over the receptive field of the FAI unit whose spike train raster plots arc shown in Figure 1. $A$, Geometric mean ( $\pm 1 \mathrm{SD}$ ) number of stimulus-evoked spikes versus stimulus velocity. $B$, Geometric mean ( \pm 1 SD) duration of stimulus-evoked activity versus stimulus velocity. $C$, Geometric mean $( \pm 1 \mathrm{SD})$ mean firing rate versus stimulus velocity. $D$, Geometric mean ( $\pm 1 \mathrm{SD}$ ) peak firing rate versus stimulus velocity. Data obtained with a brown brush (open squares) and a relatively stiffer white brush (open circles) are illustrated. All axes are scaled logarithmically.

on the neural response in a manner similar to that of the previous investigators, we extensively studied the relationship between the mean firing rate and stimulus velocity. First, each of the 152 runs of data that contained the neural response to more than one stimulus velocity was divided into two datasets (one for each opposing direction of motion). Linear regression techniques were then applied to the logarithms of the mean firing rate (MFR) and velocity (VEL) to obtain the best fit to the model:

$$
\mathrm{MFR}=c \cdot(\mathrm{VEL}-a)^{n},
$$

where $c$ is the multiplicative constant, namely, the predicted MFR at $1.0 \mathrm{~cm} / \mathrm{sec}$ when $a$ is zero, $a$ is a threshold constant, and $n$ is an exponent reflecting the sensitivity of MFR to changes in stimulus velocity (see Essick et al., 199 l b). Estimates of the stimulus-evoked MFRs were obtained from only the abovebackground component of the discharge ( 9 of the 17 spontaneously active units exhibited biphasic responses, that is, a discharge increase followed by a decrease). Cases for which estimates of the mean firing rate could not be obtained at the lowest or highest velocities of stimulus motion were not included in the analyses (i.e., when less than two spikes were evoked by the stimuli). Moreover, for 29 of the 304 datasets, a fit could not be obtained because the stimulus conditions were such that less than two spikes per stimulus were evoked at all velocities of motion. For most datasets (viz., 262 of the 275), the estimate of the parameter $a$ was not significantly different than zero at the 0.05 level; accordingly, estimates of $c, n$, and $R^{2}$ were recalculated with this parameter of the model removed:

$$
\operatorname{MFR}=c \cdot \text { VEL }^{n} .
$$

Overall, a power function provided an excellent description of the MFR-versus-velocity relationship (the median $R^{2}$ was 0.988 ; the tenth percentile was 0.938 ). To illustrate, five datasets that vary in goodness-of-fit to a power function are shown in Figure 4.

Since some units responded poorly to the slowest movement, the velocity $v_{i}$, below which less than one impulse per second (imp/sec) would have been evoked, was estimated as follows. By setting MFR to $1.0 \mathrm{imp} / \mathrm{sec}$, and representing the velocity at which this discharge rate is evoked by $v_{i}$, the logarithmic transformation of Equation 1 becomes

$$
\begin{aligned}
& \log 10(1)=0=\log _{10}(c)+n \cdot \log _{10}\left(v_{i}-a\right) \\
& \Rightarrow v_{i}=10^{-\log 10(c) / n}+a .
\end{aligned}
$$

Because the mean firing rate saturated at velocities less than the highest studied for only 9 of the 70 mechanoreceptors (6 FA units and 3 SAII units), an upper velocity above which no information about changes in velocity was made available, was not calculated.

\section{Effect of velocity on mean neural response cannot be attributed to variations in the averaged force applied across the $R F$}

In previous animal studies, a soft brush was employed as the stimulus instrument and delivered about $5 \mathrm{gm}$ wt of force at all velocities (Whitsel et al., 1972; Franzén et al., 1984; Greenspan, 1992). This conclusion was derived from measurements obtained from a transducer positioned to emulate the skin field. In the present experiments, the mechanical interface between the shaft of the motor and the brush was equipped with two 


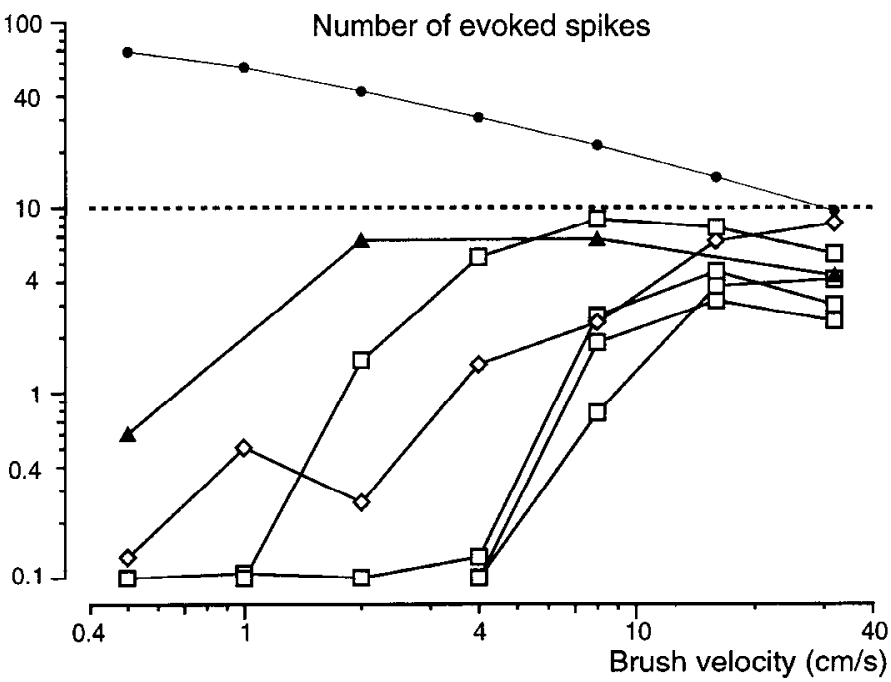

Figure 3. Geometric mean number of stimulus-evoked spikes versus stimulus velocity for the six FA mechanoreceptors that responded most poorly to the slowest velocities of stimulus motion (see curves below horizontal dashed line). Four units were located in the median nerve (open squares); one unit, in the radial nerve (open diamond); and one unit, in the inferior alveolar nerve (solid triangle). These units exhibited the steepest mean firing rate-versus-velocity relationships. For comparison, data from Figure $2 A$ (solid circles) have been replotted.

orthogonally positioned pairs of strain gauges to empirically measure the forces applied both normally and tangentially to the skin surface during each stimulus period. Consistent with the measurements made by other investigators, the brown brush delivered normal forces within the vicinity of $5 \mathrm{gm} \mathrm{wt}$ (see Materials and Methods). Careful analysis, however, revealed small but statistically significant $(p<0.05)$ variations in the normal (tangential) forces applied across the RF in 60\% (80\%) of the 197 data sets for which force data were available. Given the reliability at which the same force was applied upon replication of the same stimulus (see Edin et al., 1994), even small variations in the forces among velocities were statistically significant. The pattern of variation in the forces varied from run to run. On the average, however, the normal force tended to decrease and the tangential force tended to increase with stimulus velocity.

One hypothetical explanation for the power function relationship between MFR and velocity of stimulus motion is that it reflected concomitant, systematic and uncontrolled variations in the forces applied across the RF. This possibility was evaluated as follows. First, for each dataset, we calculated Pearson's correlation coefficient for the associations between (1) log MFR and $\log$ VEL (hereafter referred to as $r_{\text {mf vs vel }}$ ), (2) log MFR and $\log$ normal force $\left(r_{\text {mfr rs nforce }}\right)$, and (3) log MFR and log tangential force $\left(r_{\text {mfr vs torce }}\right)$. Given the null hypothesis that velocity-dependent variations in the mean firing rate were due solely to concomitant variations in the normal force applied to the RF, then $r_{\text {mfr vs nforce }}$ should equal $r_{\text {mfr vs vel. }}$ Similarly, given the null hypothesis for the tangential force, then $r_{\text {mfr vs trace }}$ should equal $r_{\text {mfr vs vel }}$. To test these hypotheses, the correlation coefficients were plotted as shown in Figure 5, $A$ and $B$. As illustrated, the magnitudes of the correlations of MFR with the normal and tangential forces not only failed to match the magnitude of the correlations of MFR with velocity, but their directions were not always the same (i.e., the correlations with force were positive for some datasets and negative for others). Thus, the empirically measured forces do not vary as a function of stimulus velocity in a manner consistent with the power function relationship between mean firing rate and velocity.

Class-and nerve-related differences in the effect of velocity on the mean neural response

For 67 of the mechanoreceptors, the MFR-versus-velocity relationship was studied with stimuli moving across the $1.4 \mathrm{~cm}$ $\times 1.4 \mathrm{~cm}$ aperture oriented parallel to the longitudinal axis of the hand or transversely across the face. With the aperture positioned in this manner, the stimuli moved proximal-to-distally and distal-to-proximally on the hand, or lateral-to-medially ( $\mathbf{L}$ $\rightarrow \mathbf{M}$ ) and medial-to-laterally $(\mathrm{M} \rightarrow \mathrm{L})$ on the face. Power functions were fit to the averaged data of 65 of the 67 mechanoreceptors. For two units, power functions could not be fit to the data because less than two spikes per stimulus, on the average, were evoked at all velocities in each of the two opposing directions of motion. Moreover, power functions could be fit to the data obtained in only one direction for two additional units.

To evaluate class- and nerve-related differences in the effect of velocity on the mean neural response, estimates of $n, c$, and $v_{i}$ were first calculated based on all the data available for each mechanoreceptor. The cumulative distributions of the values are shown in Figure 6 . Three major observations are readily made upon inspection of these plots. First, the FA units appeared to exhibit the greatest sensitivity to changes in stimulus velocity (in column of plots to the left, note that the solid curves are displaced to the right of the dashed and dotted curves). Second, the velocity at which the units first responded to the moving stimuli appeared to be higher for the FA than for the SA units (in column of plots to the right, note that the solid curves are displaced to the right of the dashed and dotted curves). And third, stimuli moving at $1.0 \mathrm{~cm} / \mathrm{sec}$ appeared to evoke the greatest responses in the mechanoreceptors of the inferior alveolar nerve (in middle column of plots, note that the curves in the bottom row are displaced to the right of those in the middle and top rows).

To statistically investigate these possibilities, the estimates of $n, v_{i}$, and $c$ were subjected to analysis of variance to determine whether there were differences among nerves or among units of different classes. The analysis confirmed that the geometric mean estimate of $n$ for the FA mechanoreceptors $(0.77)$ statistically exceeded the estimates for the SAI (mean $n=0.49$ ) and SAII (mean $n=0.45$ ) mechanoreceptors $(p<0.002)$. This finding is interpreted to imply that the population of FA units was more sensitive to changes in stimulus velocity than either population of SA mechanoreceptors. To illustrate, a 4.4-fold increase in stimulus velocity was required to double the population response of the SA units. In contrast, stimulus velocity had to increase only 2.5 times to double the population response of the FA mechanoreceptors.

Analysis of the parameter $v_{i}$ confirmed that the FA units were less responsive, on the average, to the lowest stimulus velocities $(p<0.0001)$. Specifically, the median (maximum) $v_{i}$ was 0.391 $(6.47), 0.002(0.395)$, and $0.004 \mathrm{~cm} / \mathrm{sec}(0.538)$ for the FA, SAI, and SAII mechanoreceptors, respectively. That these differences between the SA and FA units indicate notably different capacities to encode information about stimulus velocity is illustrated by Figure 7 . This figure provides a scatter plot of the estimates of $n$ versus the estimates of $v_{i}$. Note that although the data points from the SA and FA units overlap, they are represented pre- 


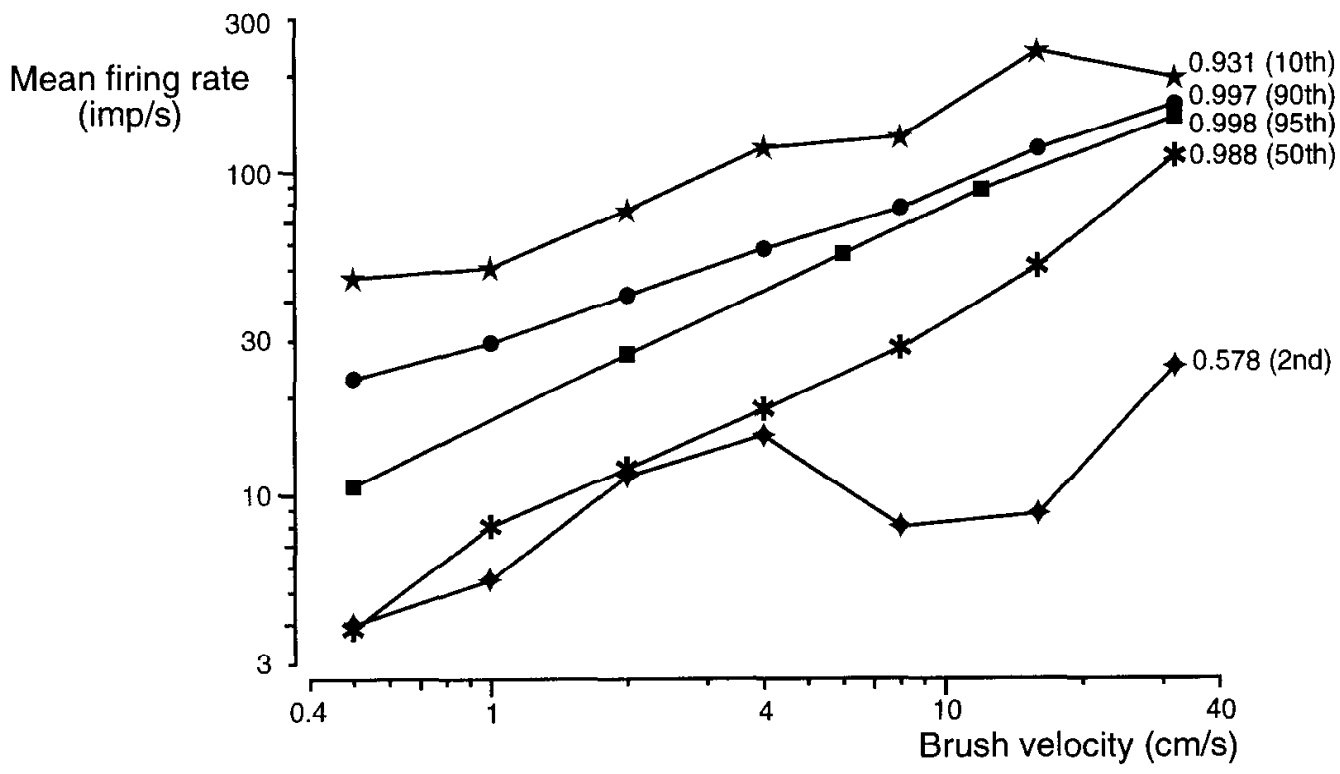

Figure 4. Five datasets are shown to illustrate the adequacy of a power function model to describe the mean firing rate-versus-velocity relationship. The degree to which each dataset fit the model is expressed by the $R^{2}$ value indicated to the right of the curve. The datasets were chosen to illustrate the poorest fits (viz., the 2 nd and 10 th percentile $R^{2}$ values), an average fit (viz., the 50 th percentile $R^{2}$ value), and the best fits (viz., the 90 th and 95 th percentile $R^{2}$ values).

dominantly at different regions on the graph. Moreover, whereas $n$ and $v_{i}$ vary independently for the SAs (see circles), this is not the case for the FAs (see triangles). Specifically, for the FAs, higher values of $v_{i}$ were associated with greater values of $n$. This suggests that the FA units which were most sensitive to changes in velocity were the least responsive of all units to the slowest moving stimuli. Considered together, these observations provide uncquivocal evidence that the SA and FA mechanoreceptors represent two different peripheral encoding processes for tactile motion.

Analysis of the estimates of the parameter $c$ confirmed that the units in the inferior alveolar nerve were, on the average, more responsive to stimuli moving at $1.0 \mathrm{~cm} / \mathrm{sec}$ than the units in the median and radial nerves $(p<0.002$; arithmetic mean $c$ was $37.3,15.5$, and 23.8 , respectively). Analyses of the predicted mean firing rates for stimuli moving at 8.0, 16.0, and $32.0 \mathrm{~cm} / \mathrm{sec}$ further revealed that the greatest and least responses were evoked in mechanoreceptors in the inferior alveolar and median nerves, respectively, over the entire range $1-32 \mathrm{~cm} / \mathrm{sec}$. At the higher velocities (viz., 16.0 and $32.0 \mathrm{~cm} / \mathrm{sec}$ ), large classrelated differences were also observed: the FA mechanorccptors exhibited the greatest responses; and the SAII mechanoreceptors, the least. Moreover, over the range $1-32 \mathrm{~cm} / \mathrm{sec}$, the mean firing rate response of the SAII mechanoreceptors varied the least among the three nerves from which recordings were made.
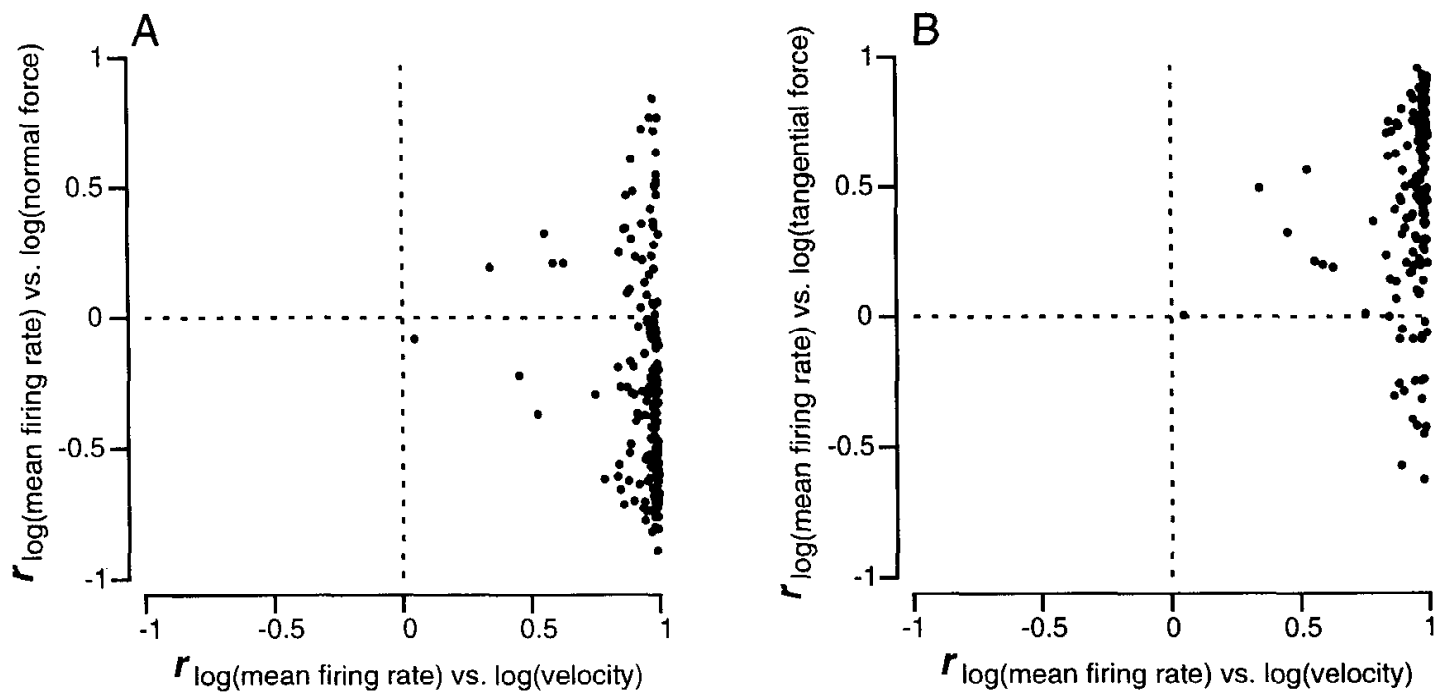

Figure 5. $A$, Correlation coefficient between $\log$ mean firing rate (MFR) and log normal force $\left(r_{\text {mfr }}\right.$ vs norce $)$ plotted as a function of the correlation coefficient between log MFR and log velocity, VEL $\left(r_{\text {mfr vs vel }}\right)$. B. Correlation coefficient between $\log$ MFR and log tangential force $\left(r_{\text {mfr vs fforce }}\right)$ plotted as a function of the correlation coefficient between $\log$ MFR and $\log$ VEL $\left(r_{\mathrm{mfr} \text { vs vel }}\right)$. 

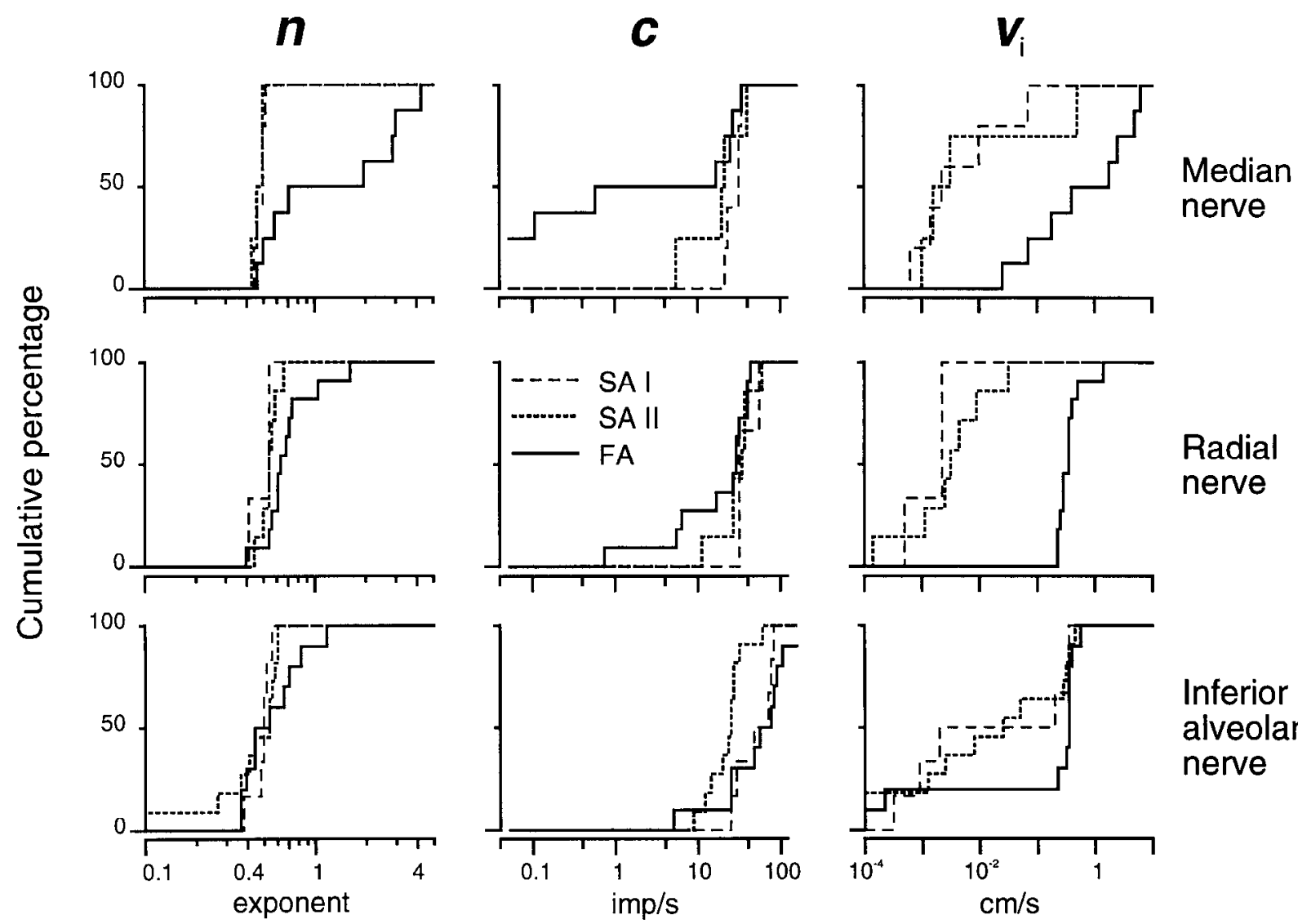

\section{Radial \\ nerve}
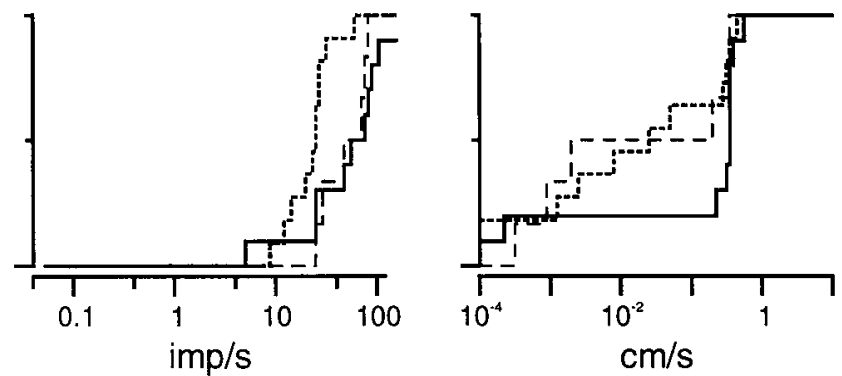

\section{Inferior alveolar nerve}

Figure 6. Cumulative distributions of the estimates of the parameters of the power function relationship between mean firing rate and stimulus velocity, $n$ (exponent of the relationship; column of plots to the left) and $c$ (predicted MFR at $1.0 \mathrm{~cm} / \mathrm{sec}$; middle column of plots), and of the parameter $v_{i}$ (predicted velocity evoking $1.0 \mathrm{imp} / \mathrm{sec}$; column of plots to the right). The top, middle, and bottom rows of plots illustrate estimates from mechanorcceptors locatcd in the median, radial, and inferior alvcolar ncrves, respectivcly. The dashed, dotted, and solid curves represent data from SAI, SAII, and FA units, respectively.

Finally, we asked to what extent the parameter $c$ could be reliably employed as a measure of the overall relative responsivity of a mechanoreceptor. That is, to what extent could one expect a mechanoreceptor that exhibited a high MFR at $1.0 \mathrm{~cm} /$

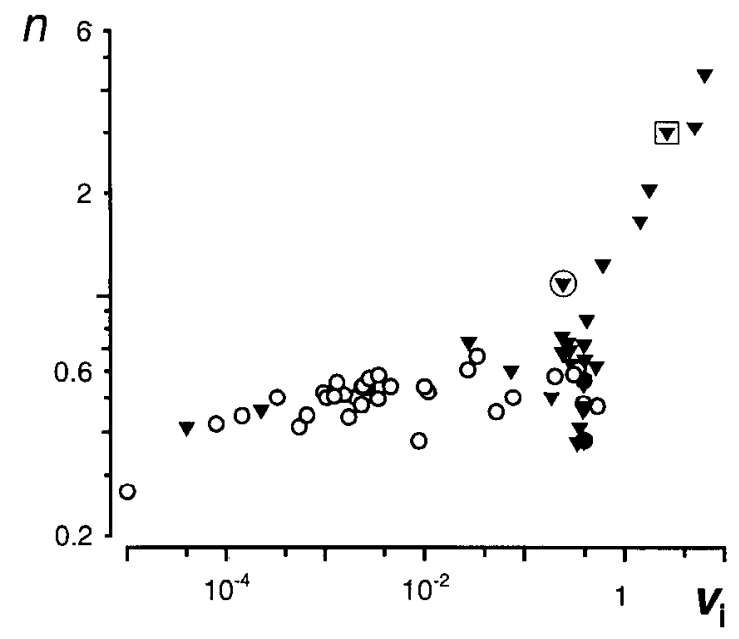

Figure 7. Scatter plot of $n$ versus $v_{i}$. Each open circle summarizes the data from one SA unit; each solid triangle, one FA unit. The data from the one FA-hair mechanoreceptor studied is encircled; from the one FAII mechanoreceptor studied, enclosed by a square. Note that both axes are scaled logarithmically. sec to exhibit relatively high MFRs at all velocities? If $c$ represents a meaningful among-unit measure of responsivity, its relationship to MFR should be well described by a line passing through the origin for every velocity of stimulus motion. This was found not to be the case due to the reciprocal nature between $c$ and $n$, particularly for the fast adapting mechanoreceptors (Fig. 8 ). As a result, the units that responded most poorly at $1.0 \mathrm{~cm} /$ sec (i.e., that provided the lowest values of $c$ ) exhibited the highest values of $n$. At the highest velocities, the firing rate of these units exceeded the firing rate of the units that were more responsive at $1.0 \mathrm{~cm} / \mathrm{sec}$. Accordingly, the usefulness of the parameter $c$ as a rclative mcasurc of responsivity to tactile motion is most appropriately limited to that observed for velocities approximating $1 \mathrm{~cm} / \mathrm{sec}$.

Class- and nerve-related differences in $\mathrm{n}, \mathrm{v}_{\mathrm{i}}$, and c cannot be attributed to variations in the averaged forces applied across the $R F$ or in the thresholds of the mechanoreceptors

One hypothetical explanation for the class-specific differences in $n$ and $v_{t}$, and regional differences in $c$ is that they reflect the presence of biases in the data. This possibility exists because (1) different brushes and brush extensions were employed to study mechanoreceptors of different nerves, (2) the different brushes and brush extension delivered different normal and tangential forces across the RF, and (3) the threshold of the mechanoreceptors located in different nerves varied significantly (Edin et al., 1994). It was possible to analyze if the class- and 


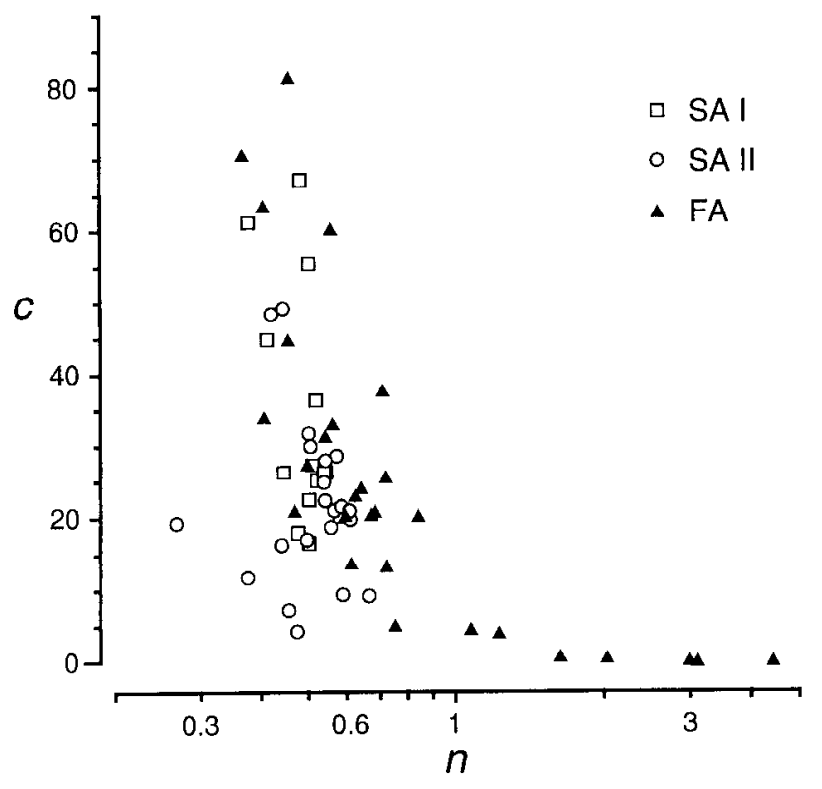

Figure 8. Scatter plot of $c$ versus $n$. Each open square summarizes the data from one SAI unit; each open circle, one SAII unit; each solid triangle, one FA unit. Note that the abscissa is scaled logarithmically.

nerve-related differences were due to these confounding factors because force data were available for all but eight of the mechanoreceptors. Specifically, the analyses of variance described in the previous section of this article was repeated with the addition of covariates to adjust for differences in the forces applied by the stimuli and in the thresholds of the mechanoreceptors. Thirty-six different covariates were explored, but only the three that accounted for the most variability in the data are described below. These three each represented a "normalized stimulus force," that is, the logarithm of the ratio of the stimulus force and the threshold force (thres) of the individual mechanoreceptor. The stimulus forces considered were the normal force (nforce), the tangential force (tforce), and the resultant force (rforce). The resultant force was calculated as the vectorial sum of the normal and tangential forces. The three covariates were labeled $\log$ (nforce: thres), $\log$ (tforce: thres), and $\log$ (rforce: thres).

Reanalysis of the exponents $n$ with the addition of one of the three covariates revealed that the estimates were affected by the normalized effective force: $12 \%$ and $8 \%$ of the total variability in $(\log ) n$ could be accounted for by variability in $\log ($ tforce : thres) and $\log$ (rforce : thres), respectively. The covariate $\log$ (nforce : thres) did not explain a statistically significant percentage (5\%) of the variability. The relationship between $n$ and each covariate is exemplified by the plot of $(\log ) n$ versus $\log$ (tforce: thres), shown in Figure $9 A$. As suggested by the plot, both linear and quadratic terms were required for the analysis of covariance. That is, $(\log ) n$ tended to decrease with $\log$ (tforce : thres) up to a value of approximately 1.5 and to remain relatively constant thereafter. Since data from the SA and FA mechanoreceptors were relatively uniformly distributed with respect to $\log$ (tforce : thres), bias due to different forces and thresholds was predicted to be minimal. In accord with this prediction, the analyses of covariance confirmed the earlier findings: The proportional increase in MFR with velocity was greater for the FA mechanoreceptors than for the SA mechanoreceptors. Due to appreciable overlap of the data from SA and FA mechanore-

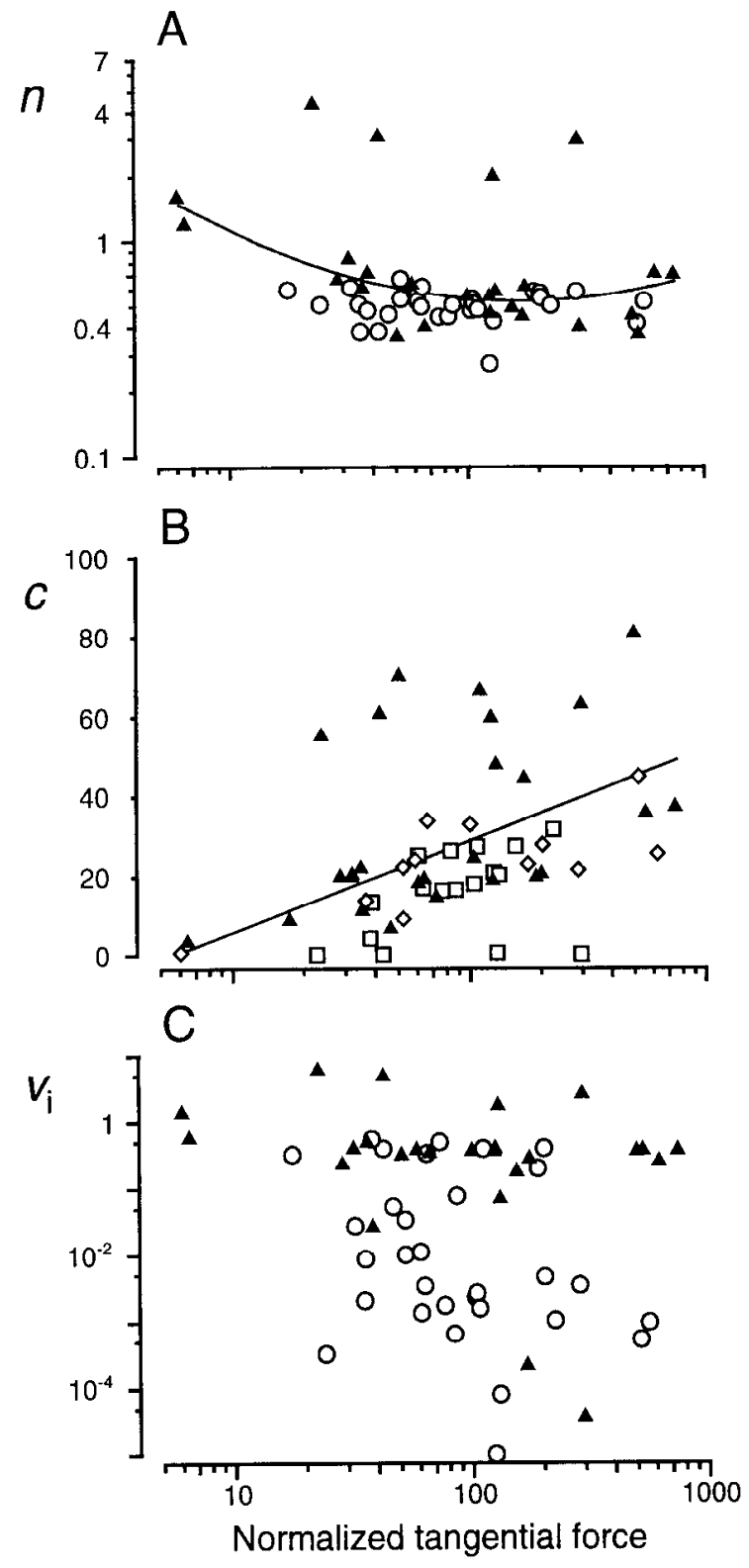

Figure 9. A, Scatter plot of estimates of $n$ versus estimates of the normalized effective tangential force. The solid curve describes the relationship between the two variables by which the mean estimates of $n$ for different nerves and classes were adjusted. Each open circle represents the data from one SA unit; each solid triangle, data from one FA unit. Both axes are scaled logarithmically. Note that the four highest values of the exponent were obtained from FA mechanoreceptors in the median nerve. Eight units were not represented in these analyses since force data were not available. $B$, Scatter plot of estimates of $c$ versus estimates of the normalized effective tangential force. Format similar to that employed for Figure $9 A$. Note that the vertical axis is scaled linearly and that the symbols depict the nerve from which the data were obtained (open squares, median nerve; open diamond, radial nerve; solid triangle, inferior alveolar nerve). $C$, Scatter plot of estimates of $v_{i}$ versus estimates of the normalized effective tangential force. Format as in Figure $9 \mathrm{~A}$.

ceptors, this finding should be interpreted as applicable to the population response rather than the response of the average (i.e., typical) unit. In addition to the effect of mechanoreceptor class, the effect of nerve attained statistically significance. This was due to reduction in the error variance afforded by the covariates. The adjusted mean estimate of $n$ for units in the median nerve 

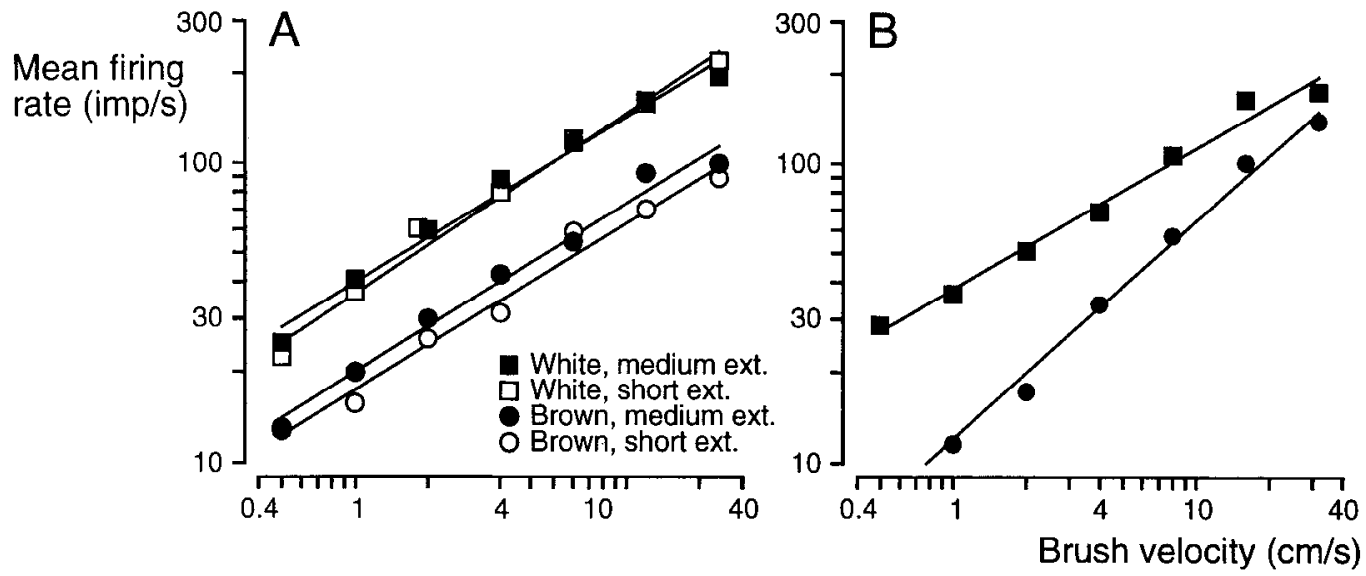

Figure 10. Geometric mean firing rate (MFR) versus stimulus velocity for 2 of 16 mechanoreceptors studied with more than one brush condition to vary the magnitude of the forces delivered across the receptive field. The trends shown by the data in $A$ were characteristics of most units; that is, MFR increased with force but the exponent of the power function (viz., slope of line) remained unchanged. A "medium extension" was defined as $2-3.5 \mathrm{~mm}$ of bristle extension through the aperture when unimpeded by the skin; a "short extension," $1-2 \mathrm{~mm}$. $R$, Data from exceptional (SAII) unit that exhibited force-dependent variation in $n$. Both axes are scaled logarithmically, and the best-fitting line has been drawn through each dataset.

exceeded that for the units in the inferior alveolar nerve $(p<$ 0.05 ). However, examination of the adjusted mean for each class revealed that this effect was largely due to exceptionally high values observed for the FA mechanoreceptors in the median nerve (see Fig. 9A).

Reanalysis of the multiplicative constants $c$ with the addition of the covariates revealed that the estimates were affected by the normalized effective force: $21 \%, 16 \%$, and $13 \%$ of the total variability in $c$ could be accounted for by variability in $\log$ (tforce : thres), $\log$ (rforce : thres), and $\log$ (nforce : thres), respectively. The relationship between $c$ and each covariate is exemplified by the plot of $c$ versus $\log$ (tforce: thres), shown in Figure $9 B$. As suggested by the plot, only a linear term was required for the analysis of covariance. That is, $c$ tended to increase linearly with increases in the normalized effective force. All three analyses of covariance confirmed that the mean estimate of $c$ for mechanoreceptors located in the inferior alveolar nerve exceeded the mean estimate for the median nerve. Given that the mean value of $c$ was greater for units in the inferior alveolar nerve than the median nerve and that the RFs of the former were mostly in hairy skin, we questioned whether units in hairy skin were, in general, more responsive to stimuli moving at $1.0 \mathrm{~cm} / \mathrm{sec}$ than units in the glabrous skin. To answer this question, the data were reanalyzed for an effect of skin type (glabrous versus hairy) rather than for an effect of nerve. The effect of skin type reached statistical significance (in all analyses, $p<0.03$ ). Specifically, the adjusted mean estimates of $c$ for units distributing to hairy skin were $65 \%$ greater than the adjusted mean estimates for units distributing to glabrous skin. These findings suggest that (1) the mechanoreceptors innervating the perioral region were more responsive to stimuli moving at $1.0 \mathrm{~cm} / \mathrm{sec}$ than those innervating the skin of the hand, and (2) mechanoreceptors with hairy skin receptive fields were, in addition, more responsive than those with receptive fields on glabrous skin. Accordingly, the mechanoreceptors with hairy perioral receptive fields were the most responsive units.

In contrast to what was found for the exponent $n$ and the constant $\mathcal{c}$, no covariate investigated accounted for a statistically significant percentage of the variability in the estimates of $v_{i}$.
The term log(tforce : thres), however, approached statistical significance $(p<0.11)$. A scatter plot of the estimates of $v_{i}$ versus $\log$ (tforce : thres) is shown in Figure $9 C$. Since the data for both classes of mechanoreceptors are relatively uniformly distributed with respect to the normalized effective forces, bias due to different forces and thresholds must be minimal. Accordingly, the finding that $v_{i}$ was higher for the FA mechanoreceptors than for the SA mechanoreceptors was accepted.

\section{Effect of averaged force on the sensitivity and responsivity of individual mechanoreceptors}

The findings of the previous section suggest that the sensitivity of the individual mechanoreceptor to changes in stimulus velocity (as assessed by the parameter $n$ ) and its responsivity to moving stimuli (as assessed by the parameter $c$ ) may vary as a function of the forces applied across the RF. Since 16 mechanoreceptors were studied with more than one brush or brush extension against the skin, this possibility was investigated.

Visual inspection suggested that the parameter $c$, but not $n$, was often affected by changes in the brush employed to obtain the data. For example, the FA mechanoreceptor whose spike train data are shown in Figure 1 was studied with two brushes at two extensions each. The data obtained with the four brush conditions and the best-fitting power functions are shown in Figure $10 \mathrm{~A}$. The ratio of the resultant forces delivered by the four brush conditions was 1.0:1.6:6.9:8.7. Importantly noted, each increment in force evoked multiplicatively greater firing rates at every velocity, which generated parallel-positioned data sets (see Fig. 10A). Thus, the parameter $c$ increased with force but the parameter $n$ did not. To evaluate the consistency of this observation among the 14 units for which force data were available, the dependency of $(\log ) n$ and $c$ on each of $\log$ (nforce: thres), $\log$ (tforce : thres), and $\log$ (rforce : thres) was statistically evaluated.

Analysis of the exponents $n$ indicated that this parameter of the power function did not vary with the different forces applied by the different brushes and brush extensions ( $p$ values $>0.29$ for normal, tangential, and resultant forces). Although this finding may appear to contradict the curvilinear relation between 
$n$ and the covariates reported in the previous section of text, this is not the case. Specifically, for the previous among-unit analyses, the range of values for each covariate exceeded $2 \mathrm{log}$ units (i.e., the maximum value exceeded 100 times the minimum value; see Fig. 9A). In contrast, use of more than one brush or brush extension to study the response of the same mechanoreceptor resulted in much smaller changes (the normal force typically varied 3.9-fold; the tangential force, 3.1 -fold; and the resultant force, 3.4 -fold). Thus, it cannot be determined from the existing data whether larger changes in force (on the order of 1-2 log units) would have affected the exponent of the power function. Data from only one of the 16 mechanoreceptors was indeed exceptional. Figure $10 B$ illustrates the MFR-versus-velocity relationship for this SAII unit whose RF was located on the dorso-radial distal forearm. The normal, tangential, and resultant forces differed only 4.1-fold, 2.7-fold, and 3.6-fold, respectively. Yet the exponent $n$ observed with the lower forces (viz., brown brush) was $54 \%$ greater than that observed with the higher forces (viz., white brush).

Finally, analysis of the multiplicative constants $c$ indicated that the responsivity of the individual mechanoreceptor to stimulus motion at $1.0 \mathrm{~cm} / \mathrm{sec}$ did increase with the forces applied by the different brushes and brush extensions ( $p$ values $<0.03$ for normal, tangential and resultant forces). Moreover, this effect was readily observed in the individual data of 11 mechanoreceptors.

\section{Directional differences in the effect of velocity on the mean neural response-opposing directions of stimulus motion}

The data for the 67 mechanoreceptors studied with stimuli moving parallel to the longitudinal axis of the hand or transversely across the face were examined to determine whether the MFRversus-velocity relationship differed for the two opposing directions of motion. Specifically, the following model was applied to the data of every run:

$$
\begin{aligned}
\log (\mathrm{MFR})= & a_{1}+a_{2} \cdot \log (\mathrm{VEL})+a_{3} \cdot \mathrm{DIR} \\
& +a_{4} \cdot \log (\mathrm{VEL}) \cdot \mathrm{DIR}+\text { error, }
\end{aligned}
$$

where DIR indicates the direction of stimulus motion, $\left(a_{1}+\right.$ $a_{3}$. DIR) equals $c$ and ( $a_{2}+a_{4}$.DIR) equals $n$ of Equation $1 \mathrm{~b}$ for both directions of motion. Type I sums-of-squares were used to evaluate the statistical significance of the estimates of $a_{1}$ to $a_{4}$ in sequential order. A level of $p \leq 0.05$ was interpreted to imply that the estimate was significantly different than zero. Accordingly, a significant estimate of $a_{3}$ was interpreted to imply that the multiplicative constant $c$ differed for the two opposing directions of motion. Similarly, a significant estimate of $a_{4}$ was interpreted to imply that the exponent $n$ differed for the two opposing directions. A total of 97 runs of data were analyzed. Only one run of data was available for 46 units and more than one run for 19 units.

It was discovered that $c$ and $n$ differed significantly by direction for $60 \%$ and $33 \%$ of the units, respectively. Both $a_{3}$ and $a_{4}$ were significant for some units. Accordingly, $72 \%$ of the mechanoreceptors exhibited statistically significant, directional differences in $c$ or $n$ or both. For $92 \%$ of the units, the effects of direction were consistently observed in every run of available data.

Second, we questioned whether either of the two opposing directions across the skin of the hand or face consistently lead to higher estimates of $n$ or $c$. Analysis of variance indicated that neither of the two opposing directions resulted in greater sen- sitivity to changes in stimulus velocity on the hand $(p>0.4)$ or on the face $(p>0.6)$. Moreover, neither of the two opposing directions resulted in greater responsivity to stimuli moving at $1.0 \mathrm{~cm} / \mathrm{sec}$ on the hand $(p>0.1)$. On the face, only one class of mechanoreceptors (the SAII units) was consistently more responsive to stimuli moving lateral-to-medially than to stimuli moving medial- to-laterally (geometric mean difference $=8.9$; $p=0.053$ ).

Third, we questioned whether the magnitude of the directional differences in $n$ and $c$ varied among mechanoreceptors of different nerves and classes. It was found that the difference in $n$ (geometric mean $=0.041 ; \log \mathrm{SD}=0.68$ ) did not vary by nerve or class $(p>0.45)$. In contrast, the difference in $c$ varied by nerve $(p<0.011)$ and by class $(p<0.007$; see Fig. $11 A)$. The difference was greater for units in the radial nerve (geometric mean $=4.9$ ) and the inferior alveolar nerve (6.5) than for units in the median nerve (1.3). In addition, the difference was greater for the SA units (geometric mean $=5.3$ and 6.0 for the type I and type II units, respectively) than for the FA units (1.4). As illustrated by Figure 11A, the FA units in the median nerve exhibited the smallest directional differences $(p<0.036)$. The responsivity of the SAs of the hairy skin of the hand and those of the skin of the perioral region was particularly sensitive to direction of movement. Considered together, these findings are interpreted to imply that many low-threshold mechanoreceptors distributing to the skin of the hand and face respond differently to opposing directions of stimulus motion across their receptive fields. However, motion across neither direction on the hand or face consistently led to power function parameters with higher values so as to suggest a "preferred" regional direction of motion.

Nerve- and class-related directional differences in responsivity cannot be attributed to directional differences in the averaged forces applied across the $R F$

One hypothetical explanation for the nerve- and class-specific directional differences in the estimates of $c$ is that they parallel differences in the forces applied across the RF. This possibility exists because changes in force most often resulted in changes in the responsivity of the units to stimulus motion (see Effect of averaged force on the sensitivity and responsivity of individual mechanoreceptors, above). Moreover, it could not be assured during data collection that precisely the same force would be delivered in each of the two opposing directions of motion. This is because we attempted to study all mechanoreceptors that were isolated, regardless of the location of their RFs. As illustrated in Edin et al. (1994), the afferents often supplied cutaneous regions that were curved or exhibited surface irregularities due to their proximity to joints, nails, the vermilion border or the corner of the mouth. In contrast to psychophysical experiments, it was not always possible to position the brushing stimulator so that the longitudinal axis of the brush was perpendicular to the test site, and the excursions of the bristles in the two opposing directions appeared "symmetrical," that is, identical except for their directions. Thus, the possibility that directional differences in force that resulted from these unavoidable and nonideal brushto-skin relationships were responsible for the significant directional differences in the power function parameters was investigated.

First, the magnitudes of the directional differences in the normal, tangential and resultant forces available for the 87 (of 97) runs were each analyzed to determine whether they could ex- 

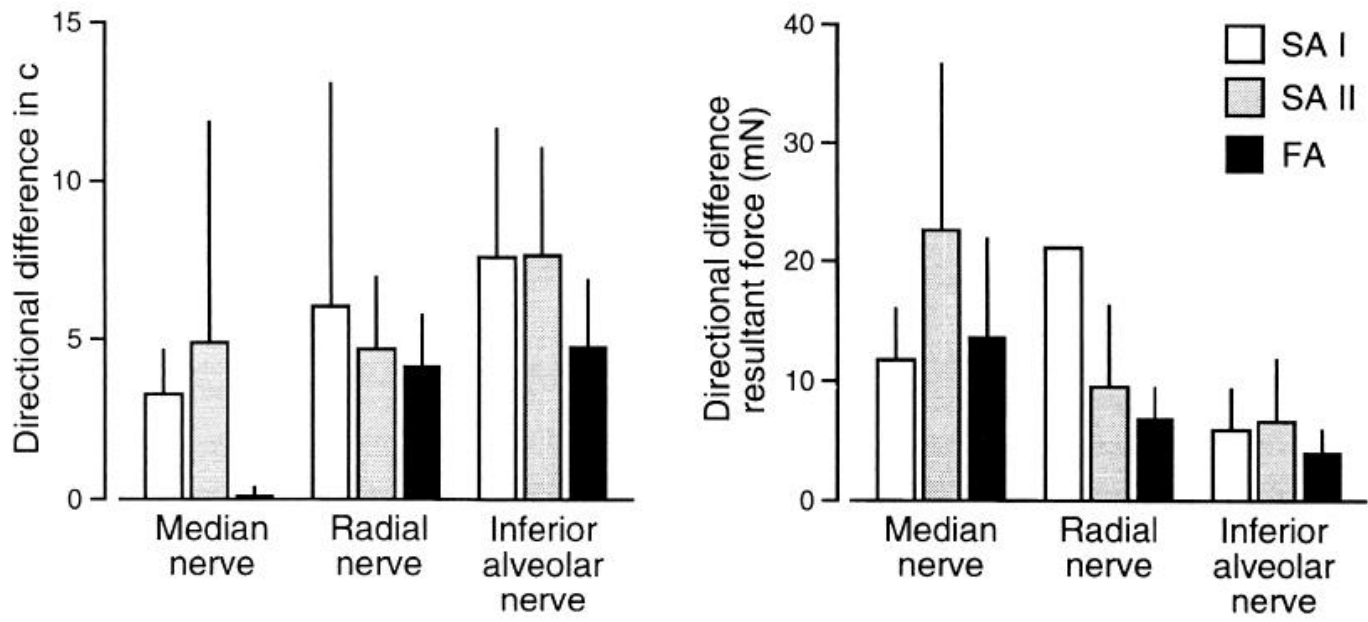

Figure 11. A, Geometric mean (+1 SE) magnitude of the directional difference in $c$ for each class of mechanoreceptor in each of the three nerves. The mean differences for the radial and inferior alveolar nerves statistically exceeded that for the median nerve. Moreover, the mean differences for the SA units exceeded that for the FA units. $B$, Geometric mean (+1 SE) magnitude of the directional difference in the resultant force for each class of mechanoreceptors in each of the three nerves. The mean difference for the median nerve statistically exceeded that for the inferior alveolar nerve.

plain the findings presented in the above section. If directional differences in force could account for the apparent greater directional sensitivity of SA units in the radial and inferior alveolar nerves, then the magnitude of the differences was predicted to be greater for these subgroups of neurons. Analysis of the data revealed that the geometric mean magnitude (and 75th percentile) of the directional difference in the normal, tangential and resultant forces were $6.0(12.5), 11.6(27.9)$, and $7.9 \mathrm{mN}$ (19.7), respectively. The magnitude of the directional differences in the tangential force did not vary among nerve $(p>0.32)$ or class $(p>0.27)$. The magnitudes of the normal and resultant

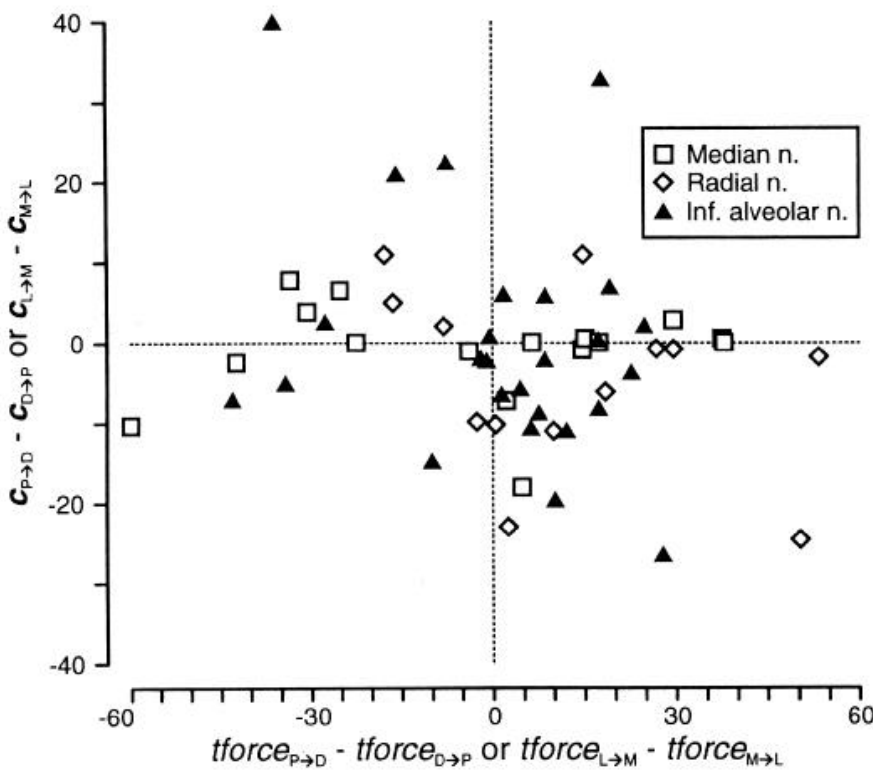

Figure 12. Directional difference in multiplicative constant $c$ plotted versus directional difference in the tangential force applied across the receptive field. Each unit is represented by a symbol to depict the nerve from which it was recorded. Note that the directional asymmetry in responsivity to stimulus motion cannot be attributed to gross differences in the tangential force applied in the two opposing directions across the RF. forces did vary among nerve $(p<0.0001$ and $p<0.05$, respectively) but not among classes of mechanoreceptors $(p>$ 0.10 and $p>0.57$, respectively). Importantly, in contrast to the prediction, the magnitude of the directional differences in these forces was greater for units in the median nerve than for units in the radial and inferior alveolar nerves (see Fig. 11B). For example, the directional difference in the resultant forces varied from $15.3 \mathrm{mN}$ (geometric mean) for the median nerve to 5.3 $\mathrm{mN}$ for the inferior alveolar nerve. Since units of the inferior alveolar nerve, unlike units of the median nerve, were only studied with the brown brush, we attributed this difference in large part to use of different brushes. In support of this conclusion, it was subsequently determined that the geometric mean (interquartile range) magnitude of directional difference in the resultant force from use of the white and brown brush was 12.2 $\mathrm{mN}(7.3-25.1)$ and $6.1 \mathrm{mN}(2.7-17.3)$, respectively.

To further investigate whether directional differences in $n$ and $c$ could be attributed to directional differences in the normal, tangential, and resultant forces applied across the RF, we examined the correlations between these two variables and their logarithms. In no case could more than $10 \%$ of the variability in the directional difference in either $n$ or $c$ be attributed to variability in the directional difference in force applied across the RFs. To illustrate, Figure 12 shows the values for the directional difference in $c$ plotted as a function of the directional differences in the tangential force. Clearly, the among-unit variability in the directional difference in the tangential force did not underlie the among-unit variability in the responsivity to the two opposing directions. Given the effect of sizable changes in stimulus force on $c$ (see Effect of averaged force on the sensitivity and responsivity of individual mechanoreceptors, above), the lack of effect observed in this analysis was attributed to the relatively small differences in the forces delivered in the two opposing directions. Specifically, the normal, tangential and resultant forces differed in the two directions on the average by only 0.18 -fold, 0.50 -fold, and 0.20 -fold, respectively. These variations in the forces are much smaller than those shown earlier in this article, to impact significantly on $c$ (i.e., 3.9-fold, 3.1-fold, and 3.4-fold, respectively). 

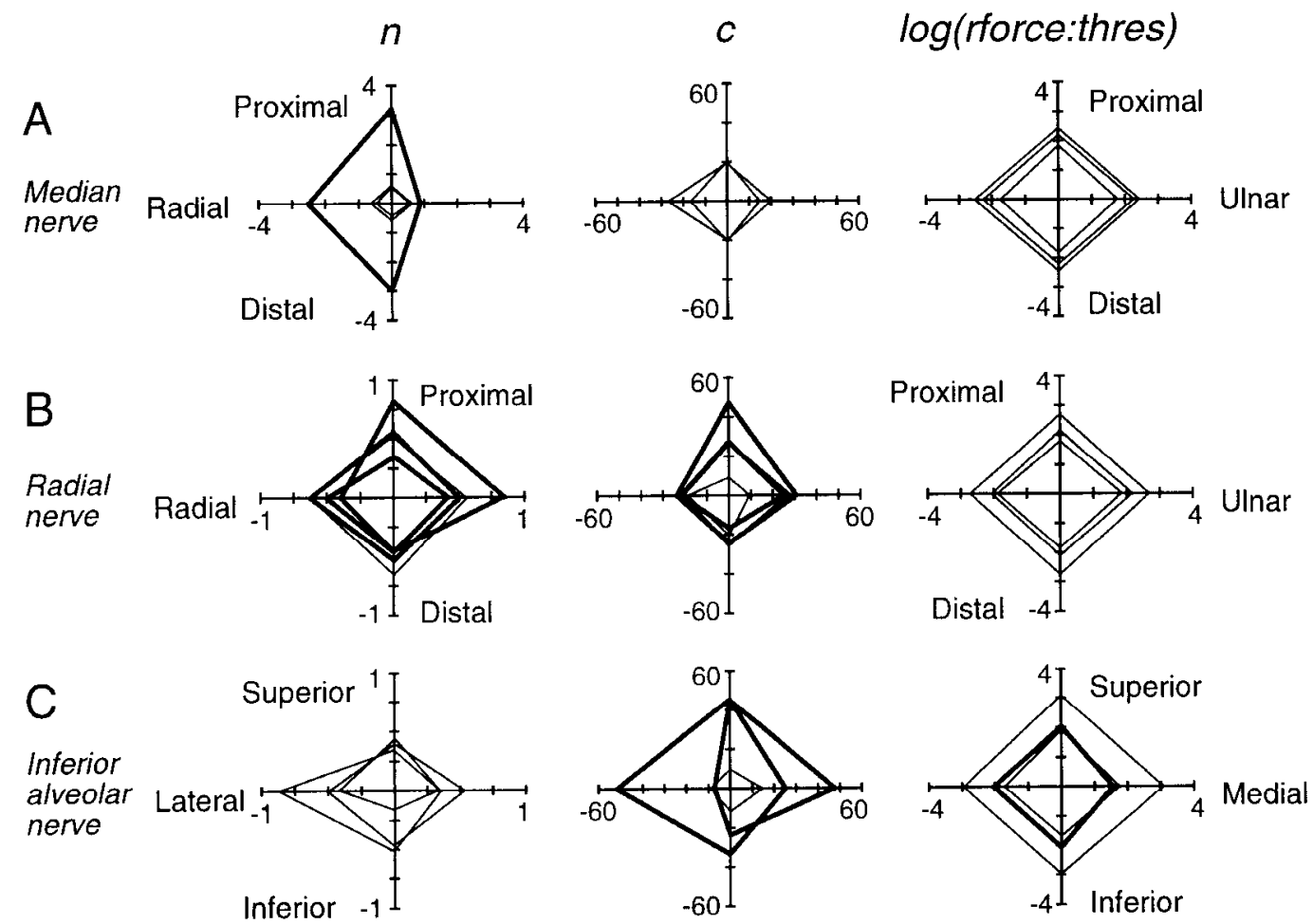

Figure 13. Polar plots for the estimates of $n, c$, and $\log$ (rforce : thres) obtained from the 10 mechanoreceptors studied with opposing directions of stimulus motion at two orthogonal orientations across the receptive field. $A$ illustrates the data from the median nerve units; $B$, the radial nerve units; and $C$, the inferior alvcolar nerve units. The data from all mechanoreceptors in each nerve are superimposed. Bold line segments connect estimates from units that differed significantly in at least one of the four directions. Force data were not available for one unit studied in the radial nerve.

\section{Directional differences in the effect of velocity on the mean neural response-orthogonal directions of stimulus motion}

In the previous two sections of text, it was shown that neither of the opposing directions of motion parallel to the longitudinal axis of the hand or transversely across the face represents a "preferred" direction of motion for mechanoreceptors inncrvating these skin regions. To extend this finding, we reexamined the data of the 10 mechanoreceptors which were, in addition, studied with stimuli moving transversely across the hand or parallel to the longitudinal axis of the face. Three of the units were located in the median nerve, four in the radial nerve, and three in the inferior alveolar nerve. Six were SA units and four were FA. More than one run of data was available for six of the units in at least one of the two orthogonal orientations.

First, repeated measures analyses of variance were performed on the estimates of $(\log ) n$ and $c$ for each nerve. It was found that five and four units exhibited dissimilar values of $n$ and $c$, respectively, at some direction(s) of stimulus motion (at $p<$ 0.05 ). To illustrate, polar plots for the estimates of $n$ (column to the left), of $c$ (middle column), and of $\log$ (rforce : thres) (column to the right) are shown in Figure 13. From the column of plots on the right, it can be seen that directional differences in $n$ and $c$ were unlikely due to grossly different forces applied across the RF: with only one exception, the resultant force did not differ significantly among directions.

Inspection of the polar plots of the estimates of $n$ and $c$ suggests that no one direction of motion over the hand or face consistently led to greater values so as to suggest a preferred regional direction of motion. This was confirmed by the statistical analyses. Specifically, $(\log ) n$ did not vary significantly with direction for the units in the median nerve $(p>0.53)$, radial nerve $(p>0.96)$, or inferior alveolar nerve $(p>0.46)$. Similarly, $c$ did not vary significantly with direction for the units in the median nerve $(p>0.66)$, radial nerve $(p>0.52)$, or inferior alveolar nerve $(p>0.83)$. Considered together, these limited data are consistent with and extend those described in the previous two sections of this article. Specifically, mechanoreceptors often exhibit small, but statistically significant, directional differences in the parameters of the power function that describes the mean firing rate-versus-velocity relationship. Accordingly, information about the orientation and direction of stimulus motion must be available in the aggregate response of the total population of mechanoreceptors activated by a moving tactile stimulus. However, no one direction on either the hand or face appears to represent a preferred regional direction of motion for all mechanoreceptors.

\section{The effect of velocity on the mean neural response is uninfluenced by the area of skin stimulated.}

In the present study most of the data was obtained with the RF centered within a $1.4 \mathrm{~cm} \times 1.4 \mathrm{~cm}$ aperture, cut into a thin Teflon plate. The dimension of the brush perpendicular to the direction of movement was approximately $1.3 \mathrm{~cm}$. As such, each brush stroke simulated a rectangularly shaped object, whose width exceeded the diameter of the RF, moving completely across the RF. Fourteen mechanoreceptors in the inferior alveolar nerve were studied not only with their receptive fields exposed to the stimuli through the $1.4 \mathrm{~cm} \times 1.4 \mathrm{~cm}$ aperture, but also through one or more aperture(s) of smaller dimensions. Four of these units were classified as SAI; five, SAII, and five, FA. In addition, one SAI mechanoreceptor in the radial nerve 


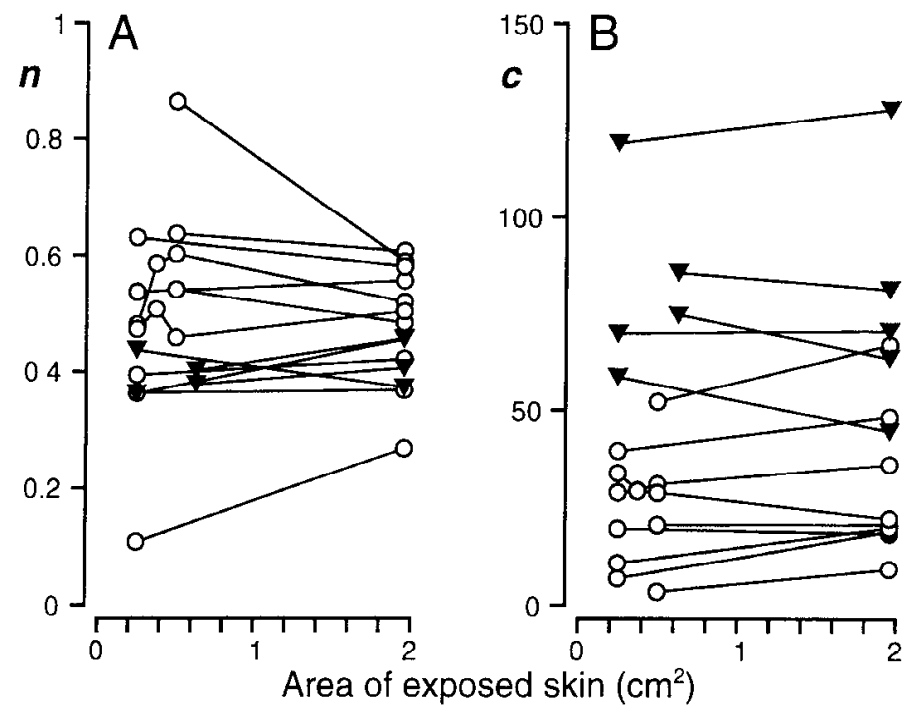

Figure 14. Plot of estimates of $n(A)$ and $c(B)$ versus the total area of skin exposed to the moving stimuli. The data from each mechanoreceptor are joined by lines. Data from slowly adapting mechanoreceptors are indicated by open circles; from fast adapting mechanoreceptors, by solid triangles. All data shown were obtained from the inferior alveolar nerve.

was stimulated without the use of an aperture. In all cases, the entire RF was exposed to the brushing stimuli (i.e., no part of the RF was ever masked by the Teflon plate). However, by reducing the aperture plate dimensions, less skin surrounding the RF was contacted by the moving bristles. The reduction in area was most often achieved by a reduction in both the length and width of skin traversed. Moreover, since the aperture plate was taped to the skin, the extent to which the RF could be displaced vertically or laterally was reduced.

To find out if changes in the area of skin exposed had an impact on the MFR-versus-velocity relationship, the estimates of $n$ and $c$ were plotted as a function of the area of skin exposed to the stimuli. As illustrated in Figure 14, the estimates were independent of the area of skin exposed. Only two mechanoreceptors provided grossly different estimates of $n$ in response to a change in the area of skin (the units whose data are represented at the very top and bottom of Fig. 14A). Repeated measures analysis of variance revealed that neither sensitivity to changes in stimulus velocity $(\log n ; p>0.58)$ nor responsivity at $1.0 \mathrm{~cm} / \mathrm{sec}(c ; p>0.48)$ was consistently impacted by this variable. This finding was confirmed for both the SA and FA units. These findings are interpreted to imply that the encoding of stimulus velocity by individual mechanoreceptors is relatively unaffected by the extent to which the skin surrounding the RF is contacted by the moving bristles.

We then questioned whether the magnitude of directional differences in $n$ and $c$ was impacted by the area of exposed skin. The geometric mean magnitude of the directional difference in $n$ and $c$ was 0.04 and 7.75 , respectively. Neither the magnitude of the directional difference in $n$ nor $c$ varied as a function of the area of skin exposed ( $p>0.18$ and 0.62 , respectively). These analyses suggest that the capacity of the individual mechanoreceptors to signal opposing directions of motion is also unaffected by the extent to which the skin surrounding the RF is contacted by the stimuli.

\section{Discussion}

Natural stimuli that move across the skin sequentially activate cutaneous low-threshold mechanoreceptors along the path of motion. As a result, information about velocity and direction of movement is provided by the successive occurrences of activity in spatially discrete sets of afferents (i.e., by an acrossfiber spatiotemporal code; Whitsel et al., 1972; see also Gardner and Palmer, 1989; Essick et al., 1991a). That the spatiotemporally distributed information is perceptually important is suggested by the deficits in direction discrimination observed in patients with peripheral nerve injuries after regeneration (e.g., see Bender et al., 1982; Essick et al., 1990). In these patients stimulus-encoding by individual afferents may be minimally impaired, but there is commonly a loss of mechanoreceptors and of somatotopic order (Dykes and Terzis, 1979; Terzis and Dykes, 1980; Hallin et al., 1981; Mackel et al., 1983; Ochs et al., 1989). Nerve-injured patients who cannot discriminatc direction of tactile motion, however, can often discern direction of skin stretch (Bender et al., 1982). In this case, information about direction may be provided by the selective activation of direction-specific populations of mechanoreceptors surrounding the skin area supplied by the injured nerve.

The analyses presented in this article were prompted by the demonstration that individual mechanoreceptors in monkeys and cats respond differentially to different velocities and directions of surface-parallel motion (Whitsel et al., 1972; Young et al., 1978; Lamb, 1983b; Franzén et al., 1984; Ray et al., 1985; Goodwin and Morley, 1987a,b; LaMotte and Srinivasan, 1987a,b). These observations suggest that information about the velocity and direction is available from the discharges of the population of mechanoreceptors activated by a moving tactile stimulus (viz., by a within-fiber code or one that does not depend on either the spatial locations of the receptive fields or their spatiotemporal sequence of activation). In the preceding companion article (Edin et al., 1994), it was argued that the patterns of discharge could not likely contribute to this hypothetical code. First, the same spatial pattern is evoked by different velocities of motion. Second, the spatial patterns do not reflect direction per se but rather mechanical events accompanying skin comprcssion, indentation, and stretch. As a result, patterns evoked by stimuli moving precisely in the same direction may differ as a result of subtle differences in skin tension or in other nonobvious differences in the manner the skin is stroked.

In this article we show that the intensity of discharge (viz., mean firing rate) is a plausible information-bearing attribute of the discharge. To summarize, a reliable monotonically increasing relationship between an afferent's mean firing rate and stimulus velocity is observed. Moreover, the relationship is reliably altered by changing the direction of stimulus motion, although the nature and magnitude of the alteration cannot be predicted on the basis of the skin region supplied or of mechanoreceptor class. In a subsequent report, we will show that the intensity of discharge sufficiently supplies relatively simple artificial neural networks with the information needed to unambiguously specify the direction and velocity of motion.

\section{The MFR response of individual cutaneous mechanoreceptors does not encode information about stimulus velocity independently of the forces applied to the $R F$}

A natural moving stimulus, such as the brush, evokes a highly complex, time-varying sequences of horizontal, vertical and shear 
stresses in the skin. As velocity is increased, the faster rate of change in stimulus components to which the receptors are particularly sensitive must effect the higher empirically observed discharge rates. The power function relationship between mean firing rate and velocity implies that a change in velocity by a constant ratio (i.e., constant number of $\log$ units) results in a change in the mean firing rate by a constant ratio (i.e., constant number of $\log$ units). In the present study it was demonstrated that an increase in the applied force most commonly results in an increase in both the number of spikes and discharge rate over the entire range of velocities employed. This observation has not been reported previously for brushing stimuli, but a similar finding has been reported for sinusoidally moving gratings applied to the glabrous digital skin of monkeys (Goodwin and Morley, 1987b).

For 14 units studied with more than one brush or brush extension, the normal force was always within the range 2.2$40.0 \mathrm{gm} \mathrm{wt}$. In contrast to the multiplicative constant $c$, the exponent $n$ of individual mechanoreceptors was, with only one exception, invariant with respect to a three- to fourfold change within this force range. Since subjects employ greater forces to discriminate the texture of rigid grooved surfaces (c.g., 17-172 gm wt, Lederman, 1974; 85-210 gm wt, Morley et al., 1983; 50-200 gm wt, Sinclair and Burton, 1991; see also Lamb, 1983a), it was concluded that the exponent does not vary over the range of force characteristic of light touch. Accordingly, an afferent's mean firing rate is anticipated to change by the same proportion in response to the same change in velocity. One interpretation is that sensitivity to change in stimulus velocity remains invariant over this range of force (an interpretation consistent with power function analyses). Proportional changes, however, do not reflect equal differences in the intensity of discharge. For example, in the present study a three- to fourfold increase in force usually resulted in a higher value of $c$. Thus, for such increments in force, greater differences in the mean firing rate were evoked by the same pair of velocities.

These findings indicate that information about stimulus force and velocity are both cncoded within the mean-firing-rate response of individual mechanoreceptors. As a result, unique combinations of higher-force/lower-velocity stimuli and lowerforce/higher-velocity stimuli evoke the same mean discharge rate. To illustrate, $105 \mathrm{imp} / \mathrm{sec}$ were evoked in the FA mechanoreceptor discussed earlier by $\mathrm{D} \rightarrow \mathrm{P}$ stimuli moving at 8 $\mathrm{cm} / \mathrm{sec}$ (with the white brush) and at $16 \mathrm{~cm} / \mathrm{sec}$ (with the brown brush; see Figs. 1, 2). The averaged resultant forces applied by these two stimulus conditions were $191 \mathrm{mN}$ and $29 \mathrm{mN}$, respectively. That information about velocity and the applied force can be disambiguated even at the single unit level is made evident upon inspection of the figures. Specifically, the higherforce/lower velocity stimulus evoked three times the number of spikes and the duration of activity was three times longer than for the lower-force/higher velocity stimulus. Similar observations were made from the other mechanoreceptors studied with more than one brush or brush extension. This leads us to conclude that attributes of the neural response other than the mean firing rate contribute importantly to an afferent's representation of movement across its receptive field.

\section{Class-related differences in the effect of velocity on the mean neural response}

In agreement with several other studies, our data show that sensitivity to change in stimulus velocity varies appreciably among cutaneous mechanoreceptors (Whitsel et al., 1972; Young et al., 1978; Franzén et al., 1984; Greenspan, 1992). Whitsel and colleagues (1972; Young et al., 1978) reported that the exponent of the best-fitting power function varied from 0.21 and 0.81 with no apparent relation to mechanoreceptor class. A comparable range of values was found by Greenspan (1992), but the values were shown to differ among classes of mechanoreceptors.

Our data exhibit striking similarities to that of Greenspan (1992). First, the sensitivity of the human SA mechanoreceptors (mean $n=0.49$ and 0.45 for SAI and SAII units, respectively) was comparable to that found in the cat (mean 0.46). Second, the highest values of $n$ were exhibited by FA mechanoreceptors. Third, both the populations of human and feline FA mechanoreceptors exhibited a broad range of sensitivities to change in stimulus velocity. In the cat, the range of sensitivities was shown to reflect the contributions of multiple subclasses of afferents which display different sensitivities to vertical-displacing, indenting stimuli (see Burgess and Perl, 1973, and Horch et al., 1977). With brushing stimuli Greenspan found that the guard-hair type $I\left(G_{1}\right)$ and fast adapting field type $I\left(F_{1}\right)$ units responded poorly to the slowest velocities, responded vigorously to the highest velocities, and exhibited the greatest exponents (mean 0.72 for $F_{1} ; 0.54$ for $G_{1}$ ). Comparatively, the $G_{2 / 1}$ and $F_{2 \prime}$ I units (both are fast adapting) responded more vigorously to the slowest velocities, responded less vigorously to the highest velocities, and exhibited lower exponents not unlike those of the SAI units (mean 0.43 for $\mathrm{G}_{2 / 1} ; 0.39$ for $\mathrm{F}_{2 / 1}$ ). A similar finding had been reported earlier by Ray et al. (1985) who demonstrated that sensitivity to the velocity of a moving air-jet stimulus varied among subclasses of feline guard-hair afferents.

The population of FA mechanoreceptors sampled in the present study clearly defined a similar continuum based on the responses to surface-parallel moving stimuli. To illustrate, many FA units exhibited exponents similar to those of the SA units (see triangles that overlap with circles in Fig. 7). Other FA units exhibited distinctly higher exponents, and these units were precisely those (1) which responded most poorly to the slowest velocities (see triangles to the far right of Fig. 7) and (2) from which only a few spikes could be evoked at any velocity (see Fig. 3).

Knibestöl (1973) found that the responses to controlled, vertically indenting stimuli in FA mechanoreceptors supplying the glabrous skin of the human hand defined a continuum with striking similarities to that of the present study. Specifically, at one end of the continuum, units were found "with a low velocity threshold, rather low sensitivity and low maximal frequency capacity;" at the other end of the continuum, units "with rather high velocity thresholds, high sensitivities and relatively high maximal frequencies" (pp 449-450). Moreover, only a few spikes were evoked in the latter at any velocity of indentation (see Fig. 8 of Knibestöl, 1973).

\section{Nerve-related differences in the effect of velocity on the mean neural response}

No prior study has addressed whether mechanoreceptors supplying different body regions exhibit different capacities to encode information about tactile motion. Although the greatest velocity sensitivity (viz., $n$ ) was exhibited by FA afferents in the median nerve, grossly comparable distributions of sensitivities were observed in all three nerves studied. In contrast, the discharge rates of the mechanoreceptors in the inferior alveolar 
nerve exceeded those of the median nerve over the entire range $1-32 \mathrm{~cm} / \mathrm{sec}$. This difference could not be explained by differences in stimulus force applied across the receptive fields and in their thresholds, but was likely due to differences in skin compliance. It was observed during the experiments that the brown brush was highly effective in indenting the perioral skin and always evoked appreciable discharge activity. In contrast, it was sometimes ineffective in evoking activity in mechanoreceptors supplying the glabrous hand. Our additional observation that the mechanoreceptors supplying hairy skin were, in general, more responsive than those supplying glabrous skin may similarly be attributed to greater strain produced in the receptive fields of the former (see Pubols, 1982).

\section{Directional differences in the effect of velocity on the mean neural response}

Previous studies of human low-threshold mechanoreceptors have only provided anecdotal reports of directionally selective (Järvilehto et al., 1976) and orientation-selective (Hagbarth et al., 1970) responses to motion across the receptive field. In this study we found that over $70 \%$ of the mechanoreceptors exhibited statistically significant directional differences in the parameters of the power function that described the MFR-versusvelocity relationship. Responsivity, as assessed by $c$, was particularly influenced by direction of motion for the SA mechanoreceptors innervating the back of the hand and the perioral region. Importantly, the increased directional selectivity of these groups of mechanoreceptors was not due to greater directional differences in the forces applied across their receptive fields. No direction of motion consistently led to estimates with higher values so as to suggest a preferred regional direction of motion.

In contrast to the paucity of human data, an appreciable amount of work regarding the directional selectivity of lowthreshold mechanoreceptors in animals has been published. Whitsel et al. (1972) reported that the response of 58 mechanoreceptive afferents supplying the monkey hairy hindlimb showed little directional preference when compared to that of many primary somatosensory cortical neurons. More recently, with the same mode of tactile stimulation employed by Whitsel et al. (1972) and in the present investigation, Greenspan (1992) observed that most mechanoreceptors supplying the feline hairy skin exhibited different response rates to opposing directions of stimulus motion along the proximo-distal axis of the hindlimb. Rigorous quantitative techniques comparable to those used in the present study were employed. Moreover, neither direction of motion was consistently preferred (but see Brown and Iggo, 1967).

Directionally selective responses (Looft, 1986; Goodwin and Morley, 1987b; LaMotte and Srinivasan, 1987a,b) and orientation-selective responses (e.g., LaMotte and Whitehouse, 1986) in monkey and feline mechanoreceptors evoked by rigid textured surfaces moved across the RF have received extensive study (see Greenspan, 1992, for review). Particularly noteworthy is the finding of Goodwin and Morley (1987b) that the directionselective responses of mechanoreceptors supplying the monkey digital skin are not artifactually generated by asymmetries in the position of the stimuli (viz., the precise area of skin contacted) in relation to the RF center. By rotating the digit over a range of $80^{\circ}$ with respect to the stimulus surface, these investigators demonstrated that neither the presence (or absence) of directional response asymmetry, nor the preferred direction of motion, was usually affected by the position of the RF center in relation to the area of skin contacted. This suggests that although the magnitude of response to a moving tactile stimulus may diminish with distance from the RF center, mechanoreceptors continue to differentially signal opposing directions of stimulus motion.

Unique patterns of discharge and different discharge intensities evoked in individual mechanoreceptors by different directions of skin stretch, as well as by different directions of real motion across the skin, have been attributed to nonisotropic skin properties and asymmetric positioning of the receptor endorgans (LaMotte and Whitehouse, 1986; Goodwin and Morley, 1987b; Edin, 1992). That skin stretch may not be necessary for evoking asymmetric directional responses in individual afferents is evidenced by use of novel modes of tactile stimulation which minimize horizontal (lateral) skin strain. Using a discrete, vertically directed air jet, Ray et al. (1985) demonstrated that opposing directions of motion across the RF of feline guardhair afferents evoke unique patterns of discharge, one being most often a "simple reversal" of the other. The detailed patterns of discharge were thought to reflect the spatial distribution of receptor terminals within the RF, and are not observed for stimulus objects that cover larger areas of the RF or apply greater forces to the skin (the air jet delivered about one-tenth of the normal force applied by a soft brush). More recently, Gardner and colleagues studied the response to Optacon-simulated movement (Gardner and Palmer, 1989). Specifically, adjacent rows of miniature probes, positioned perpendicularly to the skin surface and separated by $\sim 1 \mathrm{~mm}$, were successively activated to emulate an edge moving across the RF. For $25 \%$ of the FAI mechanoreceptors, an additional spike was evoked in one of the two opposing directions studied. These subtle differences were attributed to the interactions that describe the manner in which information from multiple terminal endings is integrated (i.e., to lateral facilitation and postspike facilitation). Importantly noted, the directional differences in the discharge reported by Ray et al. (1985) and Gardner and Palmer (1989) result in no and relatively minor directional differences, respectively, in the mean firing rate compared to those obscrved with stimuli that impart notable lateral forces to the skin, such as a brush.

\section{The effect of velocity on the mean neural response is uninfluenced by the area of skin stimulated}

Edin and Abbs (1991; Edin, 1992) recently demonstrated that both fast and slowly adapting mechanoreceptors in human hairy skin respond to minute degrees of skin stretch up to $8 \mathrm{~cm}$ from the RF. This suggests that a relatively large population of afferents is likely stimulated by natural objects moving across the skin. In all published psychophysical studies conducted with a moving brush, however, the skin field tested has been restricted by an aperture plate similar to those employed in the work reported in this paper. Although the effect on subjects' percepts of stimulus velocity is unclear (e.g., see Essick et al., 1991b), changes in the length/width of skin contacted have been shown to have a profound effect on percepts of stimulus direction (Essick and Whitsel, 1985b; Essick et al., 1990, 1991a).

The experiments reported in this and the companion paper (Edin et al., 1994) during which the aperture size was reduced sought to determine the effect of the restriction on the individual afferent's stimulus-encoding properties. In the companion paper, it was shown that reducing the aperture size had either little effect on the stimulus-evoked discharge or altered those components evoked by the brush when in contact only with the skin 
surrounding the RF. In this article, it is shown that minimizing and restricting movements of the skin surrounding the RF do not alter either the estimates of $n$ and $c$ or directional differences in $n$ and $c$. This suggests that when moving stimuli are delivered directly to the RF, the extent to which the stimulation extends beyond the RF boundaries has a negligible effect on the individual afferent's stimulus-encoding properties. These findings importantly imply that changes in psychophysical performance observed with brushing stimuli delivered through apertures of different sizes are not due to alterations in the stimulus-encoding properties of the individual afferents. In a subsequent report, we will compare the capacities of individual mechanoreceptors and human subjects to discriminate moving tactile stimuli that vary in velocity and in direction. Analyses will be employed to further clarify the contribution of the responses evoked in individual afferents to hypothetical population-encoding schemes for moving tactile stimuli in humans.

\section{References}

Bender MB, Stacy C, Cohen J (1982) Agraphesthesia: a disorder of directional cutaneous kinesthesia or a disorientation in cutaneous space. J Neurol Sci 53:531-555.

Brown AG, Iggo A (1967) A quantitative study of cutaneous receptors and afferent fibres in the cat and rabbit. J Physiol (Lond) 193:707733.

Burgess PR, Perl ER (1973) Cutaneous mechanoreceptors and nociceptors. In: Handbook of sensory physiology. Somatosensory system, Vol 2 (Iggo A, ed), pp 29-78. Berlin: Springer.

Dykes RW, Terzis JK (1979) Reinnervation of glabrous skin in baboons: properties of cutaneous mechanoreceptors subsequent to nerve crush. J Neurophysiol 42:1461-1478.

Edin BB (1992) Quantitative analysis of static strain sensitivity in human mechanoreceptors from hairy skin. J Neurophysiol 67:1 1051113 .

Edin BB, Abbs JH (1991) Finger movement responses of cutaneous mechanoreceptors in the dorsal skin of the human hand. J Neurophysiol 65:657-670.

Edin BB, Bäckström PA, Bäckström LO (1988) Single unit retrieval in microneurography: a microprocessor-based device controlled by an operator. J Neurosci Methods 24:137-144.

Edin BB, Essick GK, Trulsson M, Olsson KA (1995) Receptor encoding of moving tactile stimuli in humans. I. Temporal pattern of discharge of individual low-threshold mechanoreceptors. J Neurosci 15:830-847.

Essick GK, Whitsel BL (1985a) Assessment of the capacity of human subjects and S-I neurons to distinguish opposing directions of motion across the skin. Brain Res Rev 10:187-212.

Essick GK, Whitsel BL (1985b) Factors influencing cutaneous directional sensitivity: a correlative psychophysical and neurophysiological investigation. Brain Res Rev 10:213-230.

Essick GK, Afferica T, Aldershof B, Nestor J, Kelly D, Whitsel B (1988) Human perioral directional sensitivity. Exp Neurol 100:506-523.

Essick GK, Dolan PJ, Turvey TA, Kelly DG, Whitsel BL (1990) Effects of trauma to the mandibular nerve on human perioral directional sensitivity. Arch Oral Biol 35:785-794.

Essick GK, Bredehoeft KR, McLaughlin DF, Szaniszlo JA (1991a) Directional sensitivity along the upper limb in humans. Somatosens Mot Res 8:13-22.

Essick GK, Franzén O, McMillian A, Whitsel B (1991b) Utilization of temporal and spatial cues to judge the velocity and traverse length of a moving tactile stimulus. In: Information processing in the somatosensory system (Franzén $O$, Westman J, eds), pp 341-352. London: Macmillan.

Franzén O, Thompson F, Whitsel B, Young M (1984) Peripheral coding mechanisms of touch velocity. In: Somatosensory mechanisms (von Euler C, Franzén O, Lindblom U, Ottoson D, eds), pp 213-226. New York: Plenum.

Gardner EP, Palmer CI (1989) Simulation of motion on the skin. I. Receptive fields and temporal frequency coding by cutaneous mechanoreceptors of OPTACON pulses delivered to the hand. J Neurophysiol 62:1410-1436.
Goodwin AW, Morley JW (1987a) Sinusoidal movement of a grating across the monkey's fingerpad: representation of grating and movement features in afferent fiber responses. J Neurosci 7:2168-2180.

Goodwin AW, Morley JW (1987b) Sinusoidal movement of a grating across the monkey's fingerpad: effect of contact angle and force of the grating on afferent fiber responses. J Neurosci 7:2192-2202.

Greenspan JD (1992) Influence of velocity and direction of surfaceparallel cutaneous stimuli on responses of mechanoreceptors in feline hairy skin. J Neurophysiol 68:876-889.

Hagbarth K-E, Hongcll A, Hallin RG, Torcbjörk HE (1970) Afferent impulses in median nerve fascicles evoked by tactile stimuli of the human hand. Brain Res 24:423-442.

Hallin RG, Wiesenfeld Z, Lindblom U (1981) Neurophysiological studies on patients with sutured median nerves: faulty sensory localization after nerve regeneration and its physiological correlates. Exp Neurol 73:90-106.

Horch KW, Tuckett RP, Burgess PR (1977) A key to the classification of cutaneous mechanoreceptors. J Invest Dermatol 69:75-82.

Järvilehto T, Hämäläinen $\mathrm{H}$, Laurinen $\mathrm{P}$ (1976) Characteristics of single mechanoreceptive fibres innervating hairy skin of the human hand. Exp Brain Res 25:45-61.

Johansson RS, Olsson KA (1976) Microelectrode recordings from human oral mechanoreceptors. Brain Res 118:307-311.

Johanssor. RS, Trulsson M, Olsson KÅ, Westberg K-G (1988) Mechanoreceptor activity from the human face and oral mucosa. Exp Brain Res 72:204-208.

Knibestöl M (1973) Stimulus-response functions of rapidly adapting mechanoreceptors in the human glabrous skin area. J Physiol (Lond) 232:427-452.

Knibestöl M, Vallbo ÅB (1970) Single unit analysis of mechanoreceptive activity from the human glabrous skin. Acta Physiol Scand 80:178-195.

Lamb GD (1983a) Tactile discrimination of textured surfaces: psychophysical performance measurements in humans. J Physiol (Lond) 338:551-565.

Lamb GD (1983b) Tactile discrimination of textured surfaces: peripheral neural coding in the monkey. J Physiol (Lond) 338:567-587.

LaMotte RH, Srinivasan MA (1987a) Tactile discrimination of shape: responses of slowly adapting mechanoreceptive afferents to a step stroked across the monkey fingerpad. J Neurosci 7:1655-1671.

LaMotte RH, Srinivasan MA (1987b) Tactile discrimination of shape: responses of rapidly adapting mechanoreceptive afferents to a step stroked across the monkey fingerpad. J Neurosci 7:1672-1681.

LaMotte RH, Whitehouse J (1986) Tactile detection of a dot on a smooth surface: peripheral neural events. J Neurophysiol 56:11091128.

Lederman SJ (1974) Tactile roughness of grooved surfaces: The touching process and effects of macro- and microsurface structure. Percept Psychophys 16:385-395.

Looft FJ (1986) Response of cat cutaneous mechanoreceptors to punctate and grating stimuli. J Neurophysiol 56:208-220.

Mackel R, Kunesch E, Waldhor F, Struppler A (1983) Reinnervation of mechanoreceptors in the human glabrous skin following peripheral nerve repair. Brain Res 268:49-65.

Meyers E, Ethington D, Ashcroft S (1958) Readability of Braille as a function of three spacing variables. J App Psychol 42:163-165.

Morley JW, Goodwin AW, Darian-Smith I (1983) Tactile discrimination of gratings. Exp Brain Res 49:291-299.

Ochs G, Schenk M, Struppler A (1989) Painful dysaesthesias following peripheral nerve injury: a clinical and electrophysiological study. Brain Res 496:228-240.

Pubols BH (1982) Factors affecting cutaneous mechanoreceptor response. I. Constant-force versus constant-displacement stimulation. J Neurophysiol 47:515-529.

Ray RH, Mallach LE, Kruger L (1985) The response of single guard and down hair mechanoreceptors to moving air-jet stimulation. Brain Res 346:333-347.

SAS Institute, Inc. (1988) The GLM procedure. In: SAS/STAT user's guide, release 603 edition, pp 549-640. Cary, NC: The Institute.

Sinclair RJ, Burton H (1991) Tactile discrimination of gratings: psychophysical and neural correlates in human and monkey. Somatosens Mot Res 8:241-248.

Terzis JK, Dykes RW (1980) Reinnervation of glabrous skin in baboons: Properties of cutaneous mechanoreceptors subsequent to nerve transection. J Neurophysiol 44:1214-1225. 
Vallbo §̊B, Hagbarth K-E (1968) Activity from skin mechanoreceptors recorded percutaneously in awake human subjects. Exp Neurol 21:270-289.

Vallbo ÅB, Johansson RS (1984) Properties of cutaneous mechanoreceptors in the human hand related to touch sensation. Hum Neurobiol 3:3-14.

Vallbo AB, Hagbarth K-E, Torebjörk HE, Wallin BG (1979) Somatosensory, proprioceptive, and sympathetic activity in human peripheral nerves. Physiol Rev 59:919-957.
Whitsel BL, Roppolo JR, Werner G (1972) Cortical information processing of stimulus motion on primate skin. J Neurophysiol 35:691717

Young M, Schreiner R, Whitsel B, Dreyer D (1978) Velocity dependence of somatosensory neuron response to moving tactile stimuli. Soc Neurosci Abstr 4:560. 